\title{
The Bouncing Motion of a Superball between a Horizontal Floor and a Vertical Wall
}

\author{
Philip J Aston ${ }^{\mathrm{a}}$ \\ Paul M Milliken ${ }^{\mathrm{a}}$ \\ $\mathrm{R}$ Shail ${ }^{\mathrm{a}}$ \\ ${ }^{a}$ Department of Mathematics \\ University of Surrey \\ Guildford \\ Surrey GU2 7XH \\ U.K.
}

Corresponding author: Philip J Aston

Email: P.Aston@surrey.ac.uk

Tel: +44 1483682631

Fax: +44 1483686071

September 2, 2010 


\begin{abstract}
In earlier work [P.J. Aston and R. Shail, Dyn. Sys. 22 (2007), pp. 291-322] the problem of the possible back and forth motion of a superball thrown spinning onto a horizontal plane was considered in detail. In this paper the problem is extended to include a vertical wall. In particular motion of the superball where it bounces alternately on the floor and the wall several times is considered. Using the same physical model as in our previous work, a nonlinear mapping is derived which relates the launch data of the $(n+1)^{\text {th }}$ floor bounce to that of the $n^{\text {th }}$. This mapping is analyzed both numerically and theoretically, and a detailed description is presented of various possible motions. Regions of initial conditions which result in a specified number of bounces against the wall are also considered.
\end{abstract}

Keywords: Bouncing superball, spin, nonlinear map, scaling invariance. 


\section{Introduction}

In a previous paper [1] two of the present authors considered in detail the mechanics of a superball bouncing back and forth on a rough horizontal plane. Reversals in direction in the horizontal motion of the ball result from the application of a tangential law of restitution at the point of impact of the ball and the plane. This concept was first introduced by Garwin [2] who used a tangential coefficient of restitution of one, which is not physically realistic. Garwin's model was modified by Cross [3] who employed a tangential coefficient of restitution $\alpha$ satisfying $0<\alpha<1$, with the horizontal velocity of the point of impact of the ball being reversed and reduced in magnitude by a factor of $\alpha$ in the impact. Further details of the physics of this model are given in [1], together with references to other theoretical and experimental work.

All who have experimented with a superball will have at sometime bounced the ball on the floor, followed by a bounce on a vertical wall. If the bounce on the wall occurs while the ball is still rising, it gives the ball some backspin, so that the direction of motion is reversed at the next bounce on the floor resulting in the ball hitting the wall a second time. With practice, the ball can be made to bounce between the floor and wall several times. Such motion is illustrated in the animations in Figs 2, 3, 5, 11. It is our purpose to give a theoretical investigation of such motions and the nonlinear mappings which they engender. To this end we establish in Section 2 the basic equations governing the model. Essentially, each journey of the ball from floor to wall to floor, assumed to take place in the same vertical plane, comprises of four events: (i) after launch from the floor the ball pursues a parabolic trajectory until it hits the wall, (ii) the rebound from the wall, (iii) the parabolic trajectory of the return journey to the floor and (iv) the impact with the floor which provides the launch data for the next excursion of the ball. The result of this analysis is the derivation of a nonlinear mapping which relates the floor launch data (linear and angular velocity components of the ball and distance from the wall) to the the same parameters after the next bounce on the floor.

In Section 3 some numerical trajectories of the nonlinear mapping are computed and examples given of motions with various numbers of floor to wall bounces. Also illustrated are the parameter spaces of initial conditions required to produce various numbers of bounces off the wall. In Section 4 a scaling invariance is introduced which rewrites the nonlinear map of Section 2 in terms of suitable canonical coordinates. This results in a three-dimensional nonlinear map, a reduction in dimension by one from the original system.

Section 5 presents some numerical results for the regions of initial conditions which will result in a given number of bounces against the wall in the canonical variables, analogous to those of Section 3 for the original variables. The next two Sections of the work analyze these numerical results in some detail, focussing on the behaviour of the mapping on two planes which comprise boundaries of the region of interest. The paper concludes by proposing a number of further questions related to the problem.

Before continuing to our analysis of the problem we have just described, we note that there are limitations to the model of the bounce of the superball that we use. It is recognised that the model introduced in [3] which was subsequently used in [1] and the present paper is an oversimplification of the physics of superball impact. Thus, the tangential coefficient of restitution $\alpha$ is known not to be constant, but to depend on factors such as the speeds, the angles of incidence and the angular velocities of the bodies in collision (see, for example,

Cross [3],[4], Labous et al [5], Dong and Moys [6], Sondergaard et al [7], although we note 
that the latter two studies were concerned with steel balls, not superballs). Further there is a number of competing models of the impact process which attempt to describe the slip and elastic restitution occurring over the area of contact of the impinging bodies. For example, Maw et al [8] study in detail the elastic displacements of colliding spheres during impact, giving particular attention (via a classical elasticity mixed boundary-value problem) to the tangential tractions generated in the collision. Stronge et al [9] model the collision by again considering the elastic impact region, which they represent by a deformable particle, the remainder of the system being treated as a rigid body. A very different approach to collision dynamics is that of Bibó et al [10], who construct a mechanical model of a ball which can exhibit the back and forward bouncing studied in [1]. Basically they consider the ball to have a rigid core attached by torsion springs to an outer casing, each component being capable of rotation about a common axis. The outer layer mimics the surface layer of the ball whilst the inner part can store energy even if the outer layer is reduced to rest during the bounce. These and other models may be able to give a more realistic description of the bouncing process. However, despite the shortcomings of the Cross model of a bounce, it has the merit of enabling progress to be made in the analytical description of the title problem of this paper, and hence is to be preferred to other models which would lead to intractable mathematical and numerical situations.

\section{The Model Equations}

We consider the motion of a solid homogeneous superball of mass $m$ and radius $a$, bouncing back and forth under gravity between a horizontal floor $(f)$ and a vertical wall $(w)$. The motion is assumed to be two-dimensional, and horizontal and vertical axes $O x$ and $O y$ are taken in the plane of motion of the centre, $C$, of the ball such that the horizontal floor is given by $y=-a,-a \leq x<\infty$ and the vertical wall by $x=-a,-a \leq y<\infty$. With this choice of coordinates, the ball centre $C$ is restricted to the positive quadrant of the plane (see Fig. 1).

Since collisions occur at two separate surfaces it is essential to formulate a clear notation for describing the progress of the ball. Let $u_{n}, v_{n}$ be the horizontal and vertical velocity components of the centre of the ball immediately after the $n^{\text {th }}$ bounce on the floor, and let $\omega_{n}$, measured positive in the direction from $O x$ to $O y$, be the angular velocity of the ball. Clearly $v_{n}>0$ by definition and we require $u_{n}<0$ in order for the ball to proceed towards the wall. Further, let $x_{n}+a$ denote the horizontal distance of the ball centre $C$ from the wall at the $n$th bounce on the floor, and $y_{n}+a$ the height of $C$ above the floor at the subsequent impact with the wall. After launch from the floor the centre of the ball describes a parabolic trajectory, and elementary mechanics shows that

$$
y_{n}=-\frac{x_{n}}{u_{n}}\left(v_{n}+\frac{g x_{n}}{2 u_{n}}\right)
$$

Immediately prior to impacting the wall the linear and angular velocity components of the ball are denoted by $u_{n}^{w_{-}}, v_{n}^{w_{-}}$and $\omega_{n}^{w_{-}}$, and immediately after the collision with the wall these components are written $u_{n}^{w_{+}}, v_{n}^{w_{+}}$and $\omega_{n}^{w_{+}}$. The ball now returns to the floor, pursuing a parabolic trajectory, and reaches it with component velocities $u_{n}^{f_{-}}, v_{n}^{f_{-}}$and $\omega_{n}^{f_{-}}$, the centre $C$ having travelled a horizontal distance $x_{n+1}$. Finally, the ball rebounds from the floor at the $(n+1)^{\text {th }}$ bounce with component velocities $u_{n+1}, v_{n+1}$ and $\omega_{n+1}$. Fig. 1 shows 
the trajectories of the centre $C$ and the linear and angular velocities of the ball immediately after three successive impacts with the floor and wall structure.

During the flight of the ball between impacts any viscous or aerodynamic effects that might arise from the motion of the ball are assumed to be small and so are ignored; it follows that in any parabolic segment of the motion, the angular and horizontal velocities remain constant. In order to describe the interaction of the superball with the wall after the $n$th bounce on the floor, we introduce normal and tangential coefficients of restitution, $e_{w}$ and $\alpha_{w}$, both in the range $(0,1)$, with a similar notation for the floor, the subscript $f$ replacing $w . e_{w}$ is the classical Newtonian coefficient whence, in the notation of the previous paragraph,

$$
u_{n}^{w_{+}}=-e_{w} u_{n}^{w_{-}}=-e_{w} u_{n}
$$

In the direction tangential to the wall it is assumed, following Cross [3] and Aston and Shail [1], that the tangential velocity of the ball at the point of contact $P_{w}$ with the wall is reversed and reduced in magnitude by a factor $\alpha_{w}$. This condition gives

$$
v_{n}^{w_{+}}-a \omega_{n}^{w_{+}}=-\alpha_{w}\left(v_{n}^{w_{-}}-a \omega_{n}^{w_{-}}\right)=-\alpha_{w}\left(v_{n}^{w_{-}}-a \omega_{n}\right),
$$

where

$$
v_{n}^{w_{-}}=v_{n}+\frac{g x_{n}}{u_{n}}
$$

A third model equation follows from the conservation of angular momentum in the bounce; taking moments about $P_{w}$, which obviates the need to introduce the impulsive friction and normal reaction at $P_{w}$, we have

$$
\frac{2}{5} m a^{2} \omega_{n}^{w_{+}}+m a v_{n}^{w_{+}}=\frac{2}{5} m a^{2} \omega_{n}+m a v_{n}^{w_{-}} .
$$

Equations (3)-(5) give

$$
\begin{aligned}
v_{n}^{w_{+}} & =\frac{2}{7}\left(1+\alpha_{w}\right) a \omega_{n}+\frac{1}{7}\left(5-2 \alpha_{w}\right)\left(v_{n}+\frac{g x_{n}}{u_{n}}\right) \\
\omega_{n}^{w_{+}} & =\frac{5}{7 a}\left(1+\alpha_{w}\right)\left(v_{n}+\frac{g x_{n}}{u_{n}}\right)+\frac{1}{7}\left(2-5 \alpha_{w}\right) \omega_{n}
\end{aligned}
$$

and (6) and (7), together with (2), furnish the launch velocities for the rebound from the wall.

We now consider the return of the ball to the floor and its rebound. The initial height of the centre of the ball above $O x$ is $y_{n}$, given by (1), and its horizontal range is $x_{n+1}$. Again, elementary mechanics of the parabolic trajectory of $C$ shows that

$$
\begin{aligned}
x_{n+1} & =-\frac{e_{w} u_{n}}{g}\left\{v_{n}^{w_{+}}+\left(\left(v_{n}^{w_{+}}\right)^{2}+2 g y_{n}\right)^{\frac{1}{2}}\right\}, \\
v_{n}^{f_{-}} & =-\left(\left(v_{n}^{w_{+}}\right)^{2}+2 g y_{n}\right)^{\frac{1}{2}}
\end{aligned}
$$

where (6) supplies $v_{n}^{w_{+}}$, and further

$$
u_{n}^{f_{-}}=u_{n}^{w_{+}}, \quad \omega_{n}^{f_{-}}=\omega_{n}^{w_{+}} .
$$


Applying the law of restitution normal to the floor and making use of (1), (6) and (9) shows that

$$
\begin{aligned}
v_{n+1} & =-e_{f} v_{n}^{f_{-}} \\
& =e_{f}\left\{\left(\frac{2}{7}\left(1+\alpha_{w}\right) a \omega_{n}+\frac{1}{7}\left(5-2 \alpha_{w}\right)\left(v_{n}+\frac{g x_{n}}{u_{n}}\right)\right)^{2}-\frac{2 g x_{n}}{u_{n}}\left(v_{n}+\frac{g x_{n}}{2 u_{n}}\right)\right\}^{\frac{1}{2}}
\end{aligned}
$$

Further, tangential restitution and conservation of angular momentum about the point of contact $P_{f}$ with the floor give equations analogous to $(3)$ and $(5)[1,3]$ given by

$$
\begin{aligned}
u_{n+1} & =\frac{1}{7}\left(5-2 \alpha_{f}\right) u_{n}^{f_{-}}-\frac{2 a}{7}\left(1+\alpha_{f}\right) \omega_{n}^{f_{-}} \\
& =\frac{1}{7}\left(5-2 \alpha_{f}\right) u_{n}^{w_{+}}-\frac{2 a}{7}\left(1+\alpha_{f}\right) \omega_{n}^{w_{+}} \\
& =-\frac{1}{7}\left(5-2 \alpha_{f}\right) e_{w} u_{n}-\frac{2 a}{7}\left(1+\alpha_{f}\right)\left\{\frac{5}{7 a}\left(1+\alpha_{w}\right)\left(v_{n}+\frac{g x_{n}}{u_{n}}\right)+\frac{1}{7}\left(2-5 \alpha_{w}\right) \omega_{n}\right\}
\end{aligned}
$$

and

$$
\begin{aligned}
\omega_{n+1} & =-\frac{5}{7 a}\left(1+\alpha_{f}\right) u_{n}^{f_{-}}+\frac{1}{7}\left(2-5 \alpha_{f}\right) \omega_{n}^{f_{-}} \\
& =-\frac{5}{7 a}\left(1+\alpha_{f}\right) u_{n}^{w_{+}}+\frac{1}{7}\left(2-5 \alpha_{f}\right) \omega_{n}^{w_{+}} \\
& =\frac{5}{7 a}\left(1+\alpha_{f}\right) e_{w} u_{n}+\frac{1}{7}\left(2-5 \alpha_{f}\right)\left\{\frac{5}{7 a}\left(1+\alpha_{w}\right)\left(v_{n}+\frac{g x_{n}}{u_{n}}\right)+\frac{1}{7}\left(2-5 \alpha_{w}\right) \omega_{n}\right\},
\end{aligned}
$$

using (10), (2) and (7). We complete the mapping from the $n^{\text {th }}$ floor bounce to the $(n+1)^{\text {th }}$ by quoting the full expression for $x_{n+1}$; from (1), (6) and (8),

$$
\begin{aligned}
x_{n+1}= & -\frac{e_{w} u_{n}}{g}\left(\frac{2}{7}\left(1+\alpha_{w}\right) a \omega_{n}+\frac{1}{7}\left(5-2 \alpha_{w}\right)\left(v_{n}+\frac{g x_{n}}{u_{n}}\right)\right. \\
& \left.+\left\{\left(\frac{2}{7}\left(1+\alpha_{w}\right) a \omega_{n}+\frac{1}{7}\left(5-2 \alpha_{w}\right)\left(v_{n}+\frac{g x_{n}}{u_{n}}\right)\right)^{2}-\frac{2 g x_{n}}{u_{n}}\left(v_{n}+\frac{g x_{n}}{2 u_{n}}\right)\right\}^{\frac{1}{2}}\right) .
\end{aligned}
$$

The mapping (11)-(14) must be supplemented by initial data. Thus we assume that the ball is launched from the floor with velocity components $u_{0}<0, v_{0}>0$ and $\omega_{0}$, with the centre $C$ at an initial position $\left(x_{0}, 0\right)$. These initial data are the output of a zeroth bounce arising from projecting the ball onto the floor. To simplify matters we assume that the floor and the wall are constructed from the same material so that $\alpha_{w}=\alpha_{f}=\alpha$ and $e_{w}=e_{f}=\beta$, say. We also non-dimensionalise by defining

$$
\tilde{v}_{n}=v_{n} / v_{0}, \quad \tilde{u}_{n}=u_{n} / v_{0}, \quad \tilde{\omega}_{n}=a \omega_{n} / v_{0} \text { and } \tilde{x}_{n}=g x_{n} / v_{0}^{2} .
$$

The mapping (11)-(14) in these non-dimensional coordinates takes the form

$$
\begin{aligned}
\tilde{x}_{n+1}=f_{1}\left(\tilde{x}_{n}, \tilde{\omega}_{n}, \tilde{u}_{n}, \tilde{v}_{n}\right)= & -\beta \tilde{u}_{n}\left(2 c_{2} \tilde{\omega}_{n}+c_{1}\left(\tilde{v}_{n}+\frac{\tilde{x}_{n}}{\tilde{u}_{n}}\right)\right. \\
& \left.+\left\{\left(2 c_{2} \tilde{\omega}_{n}+c_{1}\left(\tilde{v}_{n}+\frac{\tilde{x}_{n}}{\tilde{u}_{n}}\right)\right)^{2}-\frac{2 \tilde{x}_{n}}{\tilde{u}_{n}}\left(\tilde{v}_{n}+\frac{\tilde{x}_{n}}{2 \tilde{u}_{n}}\right)\right\}^{\frac{1}{2}}\right)(1
\end{aligned}
$$




$$
\begin{aligned}
& \tilde{\omega}_{n+1}=f_{2}\left(\tilde{x}_{n}, \tilde{\omega}_{n}, \tilde{u}_{n}, \tilde{v}_{n}\right)=5 \beta c_{2} \tilde{u}_{n}+5 c_{2} c_{3}\left(\tilde{v}_{n}+\frac{\tilde{x}_{n}}{\tilde{u}_{n}}\right)+c_{3}^{2} \tilde{\omega}_{n} \\
& \tilde{u}_{n+1}=f_{3}\left(\tilde{x}_{n}, \tilde{\omega}_{n}, \tilde{u}_{n}, \tilde{v}_{n}\right)=-\beta c_{1} \tilde{u}_{n}-10 c_{2}^{2}\left(\tilde{v}_{n}+\frac{\tilde{x}_{n}}{\tilde{u}_{n}}\right)-2 c_{2} c_{3} \tilde{\omega}_{n} \\
& \tilde{v}_{n+1}=f_{4}\left(\tilde{x}_{n}, \tilde{\omega}_{n}, \tilde{u}_{n}, \tilde{v}_{n}\right)=\beta\left\{\left(2 c_{2} \tilde{\omega}_{n}+c_{1}\left(\tilde{v}_{n}+\frac{\tilde{x}_{n}}{\tilde{u}_{n}}\right)\right)^{2}-\frac{2 \tilde{x}_{n}}{\tilde{u}_{n}}\left(\tilde{v}_{n}+\frac{\tilde{x}_{n}}{2 \tilde{u}_{n}}\right)\right\}^{\frac{1}{2}},
\end{aligned}
$$

where

$$
c_{1}=\frac{1}{7}(5-2 \alpha), \quad c_{2}=\frac{1}{7}(1+\alpha), \quad c_{3}=\frac{1}{7}(2-5 \alpha) .
$$

Note that $c_{1}, c_{2}>0$ for all $0<\alpha<1$ but that $c_{3}$ may be positive, negative or zero depending on the value of $\alpha$. Later, we will also make use of the relation

$$
10 c_{2}^{2}-c_{1} c_{3}=\alpha
$$

The formulation of the problem ensures that $\tilde{v}_{n}>0$ for all $n$ since the ball bounces off the floor in the upward direction. In addition, we require three further constraints for the iterates to be valid, given by

$$
\tilde{u}_{n}<0, \quad \tilde{x}_{n}>0, \quad \tilde{x}_{n}+2 \tilde{u}_{n} \tilde{v}_{n}<0 .
$$

The first of these ensures that the ball is moving towards the wall after the bounce and also implies that $\tilde{x}_{n+1}>0$, the second is a physical constraint, since we require the bounce to occur at a positive distance from the wall, while the third is required for the ball to hit the wall before returning to the floor (i.e. $y_{n}>0$ in (1)). We note that the first condition of (20) is necessary for the third condition of (20) to hold, assuming that $v_{n}, x_{n}>0$. If the first or third of the conditions (20) is violated, then the ball will next bounce again on the floor, not the wall, and the process breaks down.

One can conceive of the limiting situation of a wall impact in which $y_{n}=0$ with the ball grazing the floor without impacting and interacting elastically with it. From (1) this occurs when $x_{n}=-2 u_{n} v_{n} / g$, but to avoid the ball immediately interacting with the floor we also need from (6) that $v_{n}^{w_{+}}>0$, i.e.

$$
\omega_{n}>\frac{(5-2 \alpha) v_{n}}{2 a(1+\alpha)}>0 .
$$

The requirement of a sufficiently positive impact angular velocity is intuitively clear, but the grazing scenario seems so unlikely that we choose to ignore it. Hence strict inequality as in the third condition of (20) will be used. It is also of interest to note that the problem in which the ball simultaneously impacts both floor and wall in the corner is indeterminate within the context of the model. Essentially there are seven unknowns in the impact, namely four impulsive force components, two at each point of contact, and the three velocity components after the bounce. Whilst seven equations, four of restitution and three moment equations, can be written down, it is found that they are not consistent. Hence the ball is unable to respond simultaneously at the two distinct points of contact.

Finally, we note that $\tilde{u}_{0}$ is the horizontal velocity immediately after the initial (zeroth) bounce. To determine the horizontal velocity immediately before this bounce, we note that

$$
\left[\begin{array}{c}
\tilde{u}_{0} \\
\tilde{\omega}_{0}
\end{array}\right]=\left[\begin{array}{rr}
c_{1} & -2 c_{2} \\
-5 c_{2} & c_{3}
\end{array}\right]\left[\begin{array}{c}
\tilde{u}_{0}^{-} \\
\tilde{\omega}_{0}^{-}
\end{array}\right],
$$


where $\tilde{u}_{0}^{-}$and $\tilde{\omega}_{0}^{-}$are the horizontal and angular velocities immediately before the bounce $[1,3]$. Inverting the matrix and using $(19)$, we find that

$$
\tilde{u}_{0}^{-}=-\frac{1}{\alpha}\left(c_{3} \tilde{u}_{0}+2 c_{2} \tilde{\omega}_{0}\right) .
$$

The ball is initially thrown towards the wall prior to the zeroth bounce if $\tilde{u}_{0}^{-}<0$ and this implies that

$$
\tilde{\omega}_{0}>-\frac{c_{3}}{2 c_{2}} \tilde{u}_{0}
$$

\section{Some Illustrative Motions}

In this section we give some numerical examples of the motions predicted by the mapping (15)-(18). We illustrate motions in which exactly $m$ successive collisions with the wall take place. It follows from (20) that for this to be the case it is required that

$$
0<\tilde{x}_{j}<-2 \tilde{u}_{j} \tilde{v}_{j} \text { for } j=0 \ldots m-1 \text {, with } \tilde{x}_{m}>-2 \tilde{u}_{m} \tilde{v}_{m} \text {. }
$$

Note that since the first condition of (20), namely $\tilde{u}_{n}<0$, is necessary for the third condition to hold, we do not need to include this as one of the constraints in (22).

Since $\tilde{v}_{0}=1$, we need consider only three initial values. Thus, we define the open set $S_{m}$ in $\left(\tilde{x}_{0}, \tilde{u}_{0}, \tilde{\omega}_{0}\right)$-parameter space for $m=1,2, \ldots$ by

$$
S_{m}=\left\{\left(\tilde{x}_{0}, \tilde{u}_{0}, \tilde{\omega}_{0}\right): 0<\tilde{x}_{j}<-2 \tilde{u}_{j} \tilde{v}_{j} \text { for } j=0, \ldots, m-1\right\}
$$

then since the ball when making its $p^{\text {th }}$ successive impact on the wall must already have made $p-1$, it follows that

$$
S_{1} \supset S_{2} \supset \ldots \supset S_{p} \supset \ldots .
$$

If the initial data $\left(\tilde{x}_{0}, \tilde{u}_{0}, \tilde{\omega}_{0}\right) \in S_{p}$, then the ball bounces off the wall at least $p$ times. For exactly $p$ bounces, $\left(\tilde{x}_{0}, \tilde{u}_{0}, \tilde{\omega}_{0}\right) \in S_{p} \backslash S_{p+1}$.

Consider first the case of a single impact with the wall. The region $S_{1}$ of parameter space is shown shaded in Fig. 2(a) for representative ranges of $\tilde{x}_{0}, \tilde{u}_{0}$ and $\tilde{\omega}_{0}$. The open set $S_{1}$ is bounded by the planes $\tilde{x}_{0}=0$ and $\tilde{x}_{0}=-2 \tilde{u}_{0}$.

In the following examples, we take the two coefficients of restitution as $\alpha=0.5$ and $\beta=0.9$, which are close to the experimental values obtained by Cross [4] and which were also used in our previous paper [1]. As an example of a trajectory with exactly one rebound from the wall, the parameters in Fig. $2(b)$ are chosen as $\tilde{x}_{0}=0.2, \tilde{u}_{0}=-0.5, \tilde{\omega}_{0}=0$ which clearly satisfy $\tilde{x}_{0}<-2 \tilde{u}_{0}$. Then, from (15)-(18) with $\tilde{v}_{0}=1$, we find that $\tilde{x}_{1}=0.546$, $\tilde{u}_{1}=-0.018, \tilde{\omega}_{1}=-0.528, \tilde{v}_{1}=0.783$ which give $\tilde{x}_{1}>-2 \tilde{u}_{1} \tilde{v}_{1}$. In physical variables suppose that $u_{0}=-5 \mathrm{~m} / \mathrm{s}$ and $v_{0}=10 \mathrm{~m} / \mathrm{s}$; then $x_{0}=v_{0}^{2} \tilde{x}_{0} / g=2 \mathrm{~m}$ and $x_{1}=5.46 \mathrm{~m}$, using the approximate value of $g=10 \mathrm{~m} / \mathrm{s}^{2}$. The ball is launched from the plane at an angle of $63.4^{\circ}$ and subsequently rebounds from the plane nearly vertically.

Consider next the situation in which the ball makes precisely two impacts with the wall, that is $\tilde{x}_{m}<-2 \tilde{u}_{m} \tilde{v}_{m}, m=0,1$ with $\tilde{x}_{2}>-\tilde{u}_{2} \tilde{v}_{2}$. The parameter space $S_{2}$ within which the initial data must lie is shown from three different aspects in Figs 3(a) through 3(c). We note that this set is "glued" to the boundary plane $\tilde{x}_{0}=0$ over quite a large region of this plane and so having the initial bounce close to the wall is clearly desirable in this case. In 
contrast, having $\tilde{x}_{0}$ too large will mean that a second bounce against the wall is impossible, although the upper bound on $\tilde{x}_{0}$ varies significantly with $\tilde{\omega}_{0}$.

We recall that the condition for the ball to be thrown towards the wall before the initial bounce is given by (21). With our value of $\alpha=0.5$, this condition becomes

$$
\tilde{\omega}_{0}>-\frac{\tilde{u}_{0}}{6} .
$$

On the scale of the axes used in Fig. 3(a), the plane defined by $\tilde{\omega}_{0}=-\tilde{u}_{0} / 6$ (which separates the two regions where the ball is thrown towards or away from the wall) is almost indistinguishable from the vertical plane $\tilde{\omega}_{0}=0$. Thus, the region with $\tilde{\omega}_{0}>0$ corresponds to the initial throw of the ball being towards the wall, but almost all of the region with $\tilde{\omega}_{0}<0$ corresponds to the initial throw being away from the wall, but with backspin on the ball so that it reverses direction and moves towards the wall after the initial bounce. We note from Fig. 3(a) that the volume of $S_{2}$ is significantly greater for negative values of $\tilde{\omega}_{0}$, corresponding to the initial throw of the ball being away from the wall, than for positive values of $\tilde{\omega}_{0}$, where the ball is initially thrown towards the wall. Thus, having a finite height wall, standing on the wrong side of it and throwing the ball over the wall with backspin is much more likely to give two bounces against the wall than standing in front of the wall.

In order to explain this result, we refer back to the work of Aston and Shail [1]. We first consider the case where the ball is initially thrown away from the wall $\left(\tilde{u}_{0}^{-}>0\right)$ but with backspin (relative to the direction of motion, i.e. $\tilde{\omega}_{0}^{-}>0$ ) to make it reverse direction at the bounce. In this case, if there is a reversal of direction, then there must also be a reversal in the spin (for $\alpha>2 / 5$; see Fig. 2 of [1]). Thus, after the initial bounce, we have $\tilde{u}_{0}<0$ and $\tilde{\omega}_{0}<0$. On the other hand, if the ball is initially thrown towards the wall, then in a similar way, it can be shown that $\tilde{\omega}_{0}$ will almost always be positive. Reverting back to the dimensional variables $\omega_{0}$ and $u_{0}$, clearly these will have the same sign as the corresponding non-dimensional variables, as just discussed.

Using (7) and (4), the spin after the bounce on the wall is given by

$$
\omega_{0}^{w_{+}}=\frac{5 c_{2}}{a} v_{0}^{w_{-}}+c_{3} \omega_{0}
$$

where $v_{0}^{w-}$ is the vertical velocity just before the bounce on the wall. We assume that the bounce on the wall occurs before the ball reaches its maximum height so that $v_{0}^{w_{-}}>0$. Now $c_{2}>0$ also and so clearly $5 c_{2} v_{0}^{w_{-}}>0$. However, $c_{3}<0$ for $\alpha=0.5$ and so when $\omega_{0}<0$, then $\omega_{0}^{w_{+}}$consists of the sum of two positive quantities, which will therefore be quite large and positive. This corresponds to the ball having a large amount of backspin after bouncing on the wall (relative to the direction of motion) and so the ball is very likely to reverse direction at the next bounce on the floor and return to hit the wall a second time. However, if $\omega_{0}>0$, then $c_{3} \omega_{0}<0$ and so $\omega_{0}^{w_{+}}$is obtained from the sum of a positive and negative quantity. While this may still be positive, corresponding to backspin, it is likely to be small in magnitude, which means that there may not be enough backspin for the next bounce on the floor to result in the reversal in direction that is required for the second hit on the wall. This explains why an initial throw away from the wall but with backspin is much more likely to result in a second hit against the wall.

To illustrate the two wall-bounce trajectory, take initial values of $\tilde{x}_{0}=0.05, \tilde{u}_{0}=-0.2$, $\tilde{\omega}_{0}=0$ which satisfy $\tilde{x}_{0}<-2 \tilde{u}_{0}$. Then, from (15)-(18), we find that $\tilde{x}_{1}=0.219, \tilde{u}_{1}=-0.242$, $\tilde{\omega}_{1}=-0.250$ and $\tilde{v}_{1}=-0.709$ with $\tilde{x}_{1}<-2 \tilde{u}_{1} \tilde{v}_{1}$. A further iteration gives $\tilde{x}_{2}=0.108$, 
$\tilde{u}_{2}=0.207, \tilde{\omega}_{2}=-0.219$ and $\tilde{v}_{2}=0.644$ with $\tilde{x}_{2}>-2 \tilde{u}_{2} \tilde{v}_{2}$. The trajectory of the ball is shown in Fig. 3(d), with the same colour coding as in Fig. 2(b). To relate the nondimensionlised motions to a physical situation, take $u_{0}=-2 \mathrm{~m} / \mathrm{s}, v_{0}=10 \mathrm{~m} / \mathrm{s}$ and $\omega_{0}=0$; then $x_{0}=0.5 \mathrm{~m}, x_{1}=2.190 \mathrm{~m}$ and $x_{2}=1.078 \mathrm{~m}$. After its second bounce on the floor the velocity components of the ball are $u_{2}=2.072 \mathrm{~m} / \mathrm{s}$ and $v_{2}=6.443 \mathrm{~m} / \mathrm{s}$ and the ball moves away from the wall.

With an increase in the number of wall impacts the parameter regions $S_{m}$ become both sparser and more contorted. Thus Fig. 4 shows the parameter regions $S_{3}, S_{4}$ and $S_{5}$, pertaining to at least three, four or five impacts with the wall respectively. A finer search procedure would give better resolution for $S_{5}$. In contrast to $S_{2}$, we note that a small region of $S_{3}$ becomes "unglued" from the boundary plane $\tilde{x}_{0}=0$ and so, in some cases, an initial bounce very close to the wall will not give rise to three impacts with the wall. This small bubble in $S_{3}$ becomes very much larger in $S_{4}$ so that an initial bounce close to the wall is unlikely to give four impacts with the wall, at least for negative values of $\tilde{\omega}_{0}$ (which is just as well as this corresponds to throwing the ball away from the wall!). An exception to this rule is the region lower down which is still attached to the $\tilde{x}_{0}=0$ plane. However, for positive values of $\tilde{\omega}_{0}$, corresponding to throwing the ball towards the wall, small values of $\tilde{u}_{0}$ and $\tilde{x}_{0}$ are essential in order to achieve four impacts with the wall.

The change in volume from $S_{2}$ to $S_{3}$ appears to be relatively small, so achieving three bounces against the wall should not be much harder than achieving two. However, there is then a very significant reduction in volume between $S_{3}$ and $S_{4}$, indicating that four bounces against the wall would be very difficult to generate, except possibly under carefully controlled laboratory conditions. Clearly the volume has shrunk still further when going to $S_{5}$, a pattern that is presumably repeated for successively higher values of $m$.

In Figs $5(a)$ and $5(b)$ we present trajectories for three and four wall impacts. Again, the same colour coding as in Fig. 2 is used. A student project has achieved three bounces against the wall experimentally [11] and the video of this can also be seen from the link in Fig. 5(a).

\section{Scaling Invariance}

The equations (15)-(18) have a scaling invariance which occurs since the whole problem can be scaled in the horizontal and vertical directions together without essentially changing the dynamics. This scaling invariance can be expressed, for any $c>0$, as

$$
\begin{aligned}
& f_{1}\left(c^{2} \tilde{x}_{n}, c \tilde{\omega}_{n}, c \tilde{u}_{n}, c \tilde{v}_{n}\right)=c^{2} f_{1}\left(\tilde{x}_{n}, \tilde{\omega}_{n}, \tilde{u}_{n}, \tilde{v}_{n}\right), \\
& f_{i}\left(c^{2} \tilde{x}_{n}, c \tilde{\omega}_{n}, c \tilde{u}_{n}, c \tilde{v}_{n}\right)=c f_{i}\left(\tilde{x}_{n}, \tilde{\omega}_{n}, \tilde{u}_{n}, \tilde{v}_{n}\right), \quad i=2,3,4 .
\end{aligned}
$$

This implies that for any orbit of (15)-(18) given by $\left(\tilde{x}_{n}, \tilde{\omega}_{n}, \tilde{u}_{n}, \tilde{v}_{n}\right), n=0,1,2, \ldots$, then $\left(c^{2} \tilde{x}_{n}, c \tilde{\omega}_{n}, c \tilde{u}_{n}, c \tilde{v}_{n}\right), n=0,1,2, \ldots$, is also an orbit for any $c>0$. This scaling is effectively eliminated by the initial condition $\tilde{v}_{0}=1$.

As a consequence of this scaling invariance, it is possible to express the equations in terms of canonical coordinates [12], where three equations depend only on three (scale-free) variables and the fourth equation involves all four variables, with the scaling acting by addition on the fourth variable. Choosing $c=1 / \tilde{v}_{n}$ in equations (23) and (24) gives

$$
f_{1}\left(\tilde{x}_{n}, \tilde{\omega}_{n}, \tilde{u}_{n}, \tilde{v}_{n}\right)=\tilde{v}_{n}^{2} f_{1}\left(\frac{\tilde{x}_{n}}{\tilde{v}_{n}^{2}}, \frac{\tilde{\omega}_{n}}{\tilde{v}_{n}}, \frac{\tilde{u}_{n}}{\tilde{v}_{n}}, 1\right),
$$




$$
f_{i}\left(\tilde{x}_{n}, \tilde{\omega}_{n}, \tilde{u}_{n}, \tilde{v}_{n}\right)=\tilde{v}_{n} f_{i}\left(\frac{\tilde{x}_{n}}{\tilde{v}_{n}^{2}}, \frac{\tilde{\omega}_{n}}{\tilde{v}_{n}}, \frac{\tilde{u}_{n}}{\tilde{v}_{n}}, 1\right), \quad i=2,3,4 .
$$

The equations (15)-(18) can then be written as

$$
\begin{aligned}
\frac{\tilde{x}_{n+1}}{\tilde{v}_{n+1}^{2}} & =\frac{f_{1}\left(\frac{\tilde{x}_{n}}{\tilde{v}_{n}^{2}}, \frac{\tilde{\omega}_{n}}{\tilde{v}_{n}}, \frac{\tilde{u}_{n}}{\tilde{v}_{n}}, 1\right)}{f_{4}\left(\frac{\tilde{x}_{n}}{\tilde{v}_{n}^{2}}, \frac{\tilde{\omega}_{n}}{\tilde{v}_{n}}, \frac{\tilde{u}_{n}}{\tilde{v}_{n}}, 1\right)}, \\
\frac{\tilde{\omega}_{n+1}}{\tilde{v}_{n+1}} & =\frac{f_{2}\left(\frac{\tilde{x}_{n}}{\tilde{v}_{n}^{2}}, \frac{\tilde{\omega}_{n}}{\tilde{v}_{n}}, \frac{\tilde{u}_{n}}{\tilde{v}_{n}}, 1\right)}{f_{4}\left(\frac{\tilde{x}_{n}}{\tilde{v}_{n}^{2}}, \frac{\tilde{\omega}_{n}}{\tilde{v}_{n}}, \frac{\tilde{u}_{n}}{\tilde{v}_{n}}, 1\right)}, \\
\frac{\tilde{u}_{n+1}}{\tilde{v}_{n+1}} & =\frac{f_{3}\left(\frac{\tilde{x}_{n}}{\tilde{v}_{n}^{2}}, \frac{\tilde{\omega}_{n}}{\tilde{v}_{n}}, \frac{\tilde{u}_{n}}{\tilde{v}_{n}}, 1\right)}{f_{4}\left(\frac{\tilde{x}_{n}}{\tilde{v}_{n}^{2}}, \frac{\tilde{\omega}_{n}}{\tilde{v}_{n}}, \frac{\tilde{u}_{n}}{\tilde{v}_{n}}, 1\right)}, \\
\log \tilde{v}_{n+1} & =\log \tilde{v}_{n}+\log f_{4}\left(\frac{\tilde{x}_{n}}{\tilde{v}_{n}^{2}}, \frac{\tilde{\omega}_{n}}{\tilde{v}_{n}}, \frac{\tilde{u}_{n}}{\tilde{v}_{n}}, 1\right),
\end{aligned}
$$

thus expressing the equations in terms of the canonical coordinates $\tilde{x}_{n} / \tilde{v}_{n}^{2}, \tilde{\omega}_{n} / \tilde{v}_{n}, \tilde{u}_{n} / \tilde{v}_{n}$ and $\log \tilde{v}_{n}$. Note that equations (25)-(27) depend only on the three scale-free coordinates.

The advantage of these coordinates is that the new variables are always well defined since $\tilde{v}_{n} \neq 0$ and so the singular behaviour associated with $\tilde{u}_{n}=0$ (which is a boundary of our region of interest since we require $\left.\tilde{u}_{n}<0\right)$ is contained in the equations. However, we find it more convenient to use a different set of canonical coordinates, given by $\tilde{x}_{n} /\left(\tilde{u}_{n} \tilde{v}_{n}\right), \tilde{\omega}_{n} / \tilde{v}_{n}$, $\tilde{u}_{n} / \tilde{v}_{n}$ and $\log \tilde{v}_{n}$. For these variables, the singularity associated with $\tilde{u}_{n}=0$ is brought into the first variable but is removed from the equations. In terms of these variables, the equations are given by

$$
\begin{aligned}
\frac{\tilde{x}_{n+1}}{\tilde{u}_{n+1} \tilde{v}_{n+1}} & =\frac{f_{1}\left(\frac{\tilde{x}_{n}}{\tilde{u}_{n} \tilde{v}_{n}} \frac{\tilde{u}_{n}}{\tilde{v}_{n}}, \frac{\tilde{\omega}_{n}}{\tilde{v}_{n}}, \frac{\tilde{u}_{n}}{\tilde{v}_{n}}, 1\right)}{f_{3}\left(\frac{\tilde{x}_{n}}{\tilde{u}_{n} \tilde{v}_{n}} \frac{\tilde{u}_{n}}{\tilde{v}_{n}}, \frac{\tilde{\omega}_{n}}{\tilde{v}_{n}}, \frac{\tilde{u}_{n}}{\tilde{v}_{n}}, 1\right) f_{4}\left(\frac{\tilde{x}_{n}}{\tilde{u}_{n} \tilde{v}_{n}} \frac{\tilde{u}_{n}}{\tilde{v}_{n}}, \frac{\tilde{\omega}_{n}}{\tilde{v}_{n}}, \frac{\tilde{u}_{n}}{\tilde{v}_{n}}, 1\right)}, \\
\frac{\tilde{\omega}_{n+1}}{\tilde{v}_{n+1}} & =\frac{f_{2}\left(\frac{\tilde{x}_{n}}{\tilde{u}_{n} \tilde{v}_{n}} \frac{\tilde{u}_{n}}{\tilde{v}_{n}}, \frac{\tilde{\omega}_{n}}{\tilde{v}_{n}}, \frac{\tilde{u}_{n}}{\tilde{v}_{n}}, 1\right)}{f_{4}\left(\frac{\tilde{x}_{n}}{\tilde{u}_{n} \tilde{v}_{n}} \frac{\tilde{u}_{n}}{\tilde{v}_{n}}, \frac{\tilde{\omega}_{n}}{\tilde{v}_{n}}, \frac{\tilde{u}_{n}}{\tilde{v}_{n}}, 1\right)}, \\
\frac{\tilde{u}_{n+1}}{\tilde{v}_{n+1}} & =\frac{f_{3}\left(\frac{\tilde{x}_{n}}{\tilde{u}_{n} \tilde{v}_{n}} \frac{\tilde{u}_{n}}{\tilde{v}_{n}}, \frac{\tilde{\omega}_{n}}{\tilde{v}_{n}}, \frac{\tilde{u}_{n}}{\tilde{v}_{n}}, 1\right)}{f_{4}\left(\frac{\tilde{x}_{n}}{\tilde{u}_{n} \tilde{v}_{n}} \frac{\tilde{u}_{n}}{\tilde{v}_{n}}, \frac{\tilde{\omega}_{n}}{\tilde{v}_{n}}, \frac{\tilde{u}_{n}}{\tilde{v}_{n}}, 1\right)}, \\
\log \tilde{v}_{n+1} & =\log \tilde{v}_{n}+\log f_{4}\left(\frac{\tilde{x}_{n}}{\tilde{u}_{n} \tilde{v}_{n}} \frac{\tilde{u}_{n}}{\tilde{v}_{n}}, \frac{\tilde{\omega}_{n}}{\tilde{v}_{n}}, \frac{\tilde{u}_{n}}{\tilde{v}_{n}}, 1\right) .
\end{aligned}
$$

We define the new coordinates

$$
X_{n}=-\frac{\tilde{x}_{n}}{\tilde{u}_{n} \tilde{v}_{n}}, \quad U_{n}=\frac{\tilde{u}_{n}}{\tilde{v}_{n}}, \quad \Omega_{n}=\frac{\tilde{\omega}_{n}}{\tilde{v}_{n}},
$$

where the minus sign in the definition of $X_{n}$ ensures that this scale-free distance function is positive (since $\tilde{u}_{n}<0$ ). We then define the new functions

$$
\begin{aligned}
F_{1}\left(X_{n}, \Omega_{n}, U_{n}\right) & =f_{1}\left(-X_{n} U_{n}, \Omega_{n}, U_{n}, 1\right) \\
& =-\beta U_{n}\left[2 c_{2} \Omega_{n}+c_{1}\left(1-X_{n}\right)+\tilde{F}\left(X_{n}, \Omega_{n}\right)\right], \\
F_{2}\left(X_{n}, \Omega_{n}, U_{n}\right) & =f_{2}\left(-X_{n} U_{n}, \Omega_{n}, U_{n}, 1\right)
\end{aligned}
$$




$$
\begin{aligned}
& =5 \beta c_{2} U_{n}+5 c_{2} c_{3}\left(1-X_{n}\right)+c_{3}^{2} \Omega_{n}, \\
F_{3}\left(X_{n}, \Omega_{n}, U_{n}\right) & =f_{3}\left(-X_{n} U_{n}, \Omega_{n}, U_{n}, 1\right) \\
& =-\beta c_{1} U_{n}-10 c_{2}^{2}\left(1-X_{n}\right)-2 c_{2} c_{3} \Omega_{n}, \\
F_{4}\left(X_{n}, \Omega_{n}, U_{n}\right) & =f_{4}\left(-X_{n} U_{n}, \Omega_{n}, U_{n}, 1\right) \\
& =\beta \tilde{F}\left(X_{n}, \Omega_{n}\right),
\end{aligned}
$$

where

$$
\tilde{F}\left(X_{n}, \Omega_{n}\right)=\sqrt{\left[2 c_{2} \Omega_{n}+c_{1}\left(1-X_{n}\right)\right]^{2}+2 X_{n}-X_{n}^{2}} .
$$

The function $F_{4}$ is independent of $U_{n}$ and therefore this variable can be omitted from its list of arguments. Equations (28)-(31) can then be written as

$$
\begin{aligned}
X_{n+1} & =G_{1}\left(X_{n}, \Omega_{n}, U_{n}\right)=-\frac{F_{1}\left(X_{n}, \Omega_{n}, U_{n}\right)}{F_{3}\left(X_{n}, \Omega_{n}, U_{n}\right) F_{4}\left(X_{n}, \Omega_{n}\right)}, \\
\Omega_{n+1} & =G_{2}\left(X_{n}, \Omega_{n}, U_{n}\right)=\frac{F_{2}\left(X_{n}, \Omega_{n}, U_{n}\right)}{F_{4}\left(X_{n}, \Omega_{n}\right)} \\
U_{n+1} & =G_{3}\left(X_{n}, \Omega_{n}, U_{n}\right)=\frac{F_{3}\left(X_{n}, \Omega_{n}, U_{n}\right)}{F_{4}\left(X_{n}, \Omega_{n}\right)} \\
\tilde{v}_{n+1} & =\tilde{v}_{n} F_{4}\left(X_{n}, \Omega_{n}\right) .
\end{aligned}
$$

Note that we prefer to work with the variable $\tilde{v}_{n}$ rather than the canonical coordinate $\log \tilde{v}_{n}$. Since the variable $\tilde{v}_{n}$ occurs only in the last of these equations, we study the three equations (38)-(40), which involve only the three variables $X_{n}, \Omega_{n}$ and $U_{n}$, in detail. The values of the fourth variable $\tilde{v}_{n}$ are then easily determined from (41).

In the new coordinates, the three conditions in (20) become

$$
U_{n}<0, \quad 0<X_{n}<2
$$

and these constraints define the region of the three-dimensional phase space that is of interest. Thus, we define the region

$$
W=\{(X, \Omega, U): U<0,0<X<2\} .
$$

If $\left(X_{n}, \Omega_{n}, U_{n}\right) \in W$ then the ball will bounce against the wall before returning to the floor. On the other hand, if an iterate leaves $W$, then the ball will not hit the wall before returning to bounce again on the floor. Note that there are no constraints on $\Omega_{n}$ as the angular velocity can be in either direction. We observe that the condition $0<X_{n}<2$ implies that $2 X_{n}-X_{n}^{2}>0$ and this ensures that $\tilde{F}\left(X_{n}, \Omega_{n}\right)$ is well defined and hence that $\tilde{v}_{n}>0$ for all $n$ from (41).

It is easily shown that $X_{n}=2$ corresponds to the ball hitting the wall and the floor simultaneously at the next bounce, while $X_{n}=1$ corresponds to the ball hitting the wall at the highest point of its parabolic trajectory. Clearly, $X_{n}$ approaching zero corresponds to the bounce of the ball on the floor getting correspondingly close to the wall.

Finally, the condition (21) for the ball to be thrown towards the wall initially is now given by

$$
\Omega_{0}>-\frac{c_{3}}{2 c_{2}} U_{0}
$$




\section{$5 \quad$ Numerical Results}

We now present numerical results for regions that will give a specified number of bounces against the wall in the new coordinates, which are analogous to those presented in Section 3. As before, we consider parameter values of $\alpha=0.5, \beta=0.9$.

Following the approach used in Section 3, we define the open set $R_{m}$ in $\left(X_{0}, \Omega_{0}, U_{0}\right)$ parameter space for $m=1,2, \ldots$ by

$$
R_{m}=\left\{\left(X_{0}, \Omega_{0}, U_{0}\right):\left(X_{j}, \Omega_{j}, U_{j}\right) \in W \text { for } j=0, \ldots, m-1\right\} .
$$

Clearly, these sets also have the inclusion property that

$$
R_{1} \supset R_{2} \supset \ldots \supset R_{p} \supset \ldots
$$

The region $R_{1}$ is defined by the inequalities $U_{0}<0$ and $0<X_{0}<2$, with no restriction on $\Omega_{0}$. The regions $R_{2}, R_{3}, R_{4}$ and $R_{5}$ are shown in Fig. 6. Finer resolution is required to obtain $R_{5}$ more accurately.

We note from the comparable numerical results in Fig. 4 that the regions $S_{i}$ all seem to connect to the line $\tilde{x}_{0}=\tilde{u}_{0}=0$. The new variable $X_{0}$ as this line is approached is given by

$$
\lim _{\tilde{u}_{0} \rightarrow 0} X_{0}=\lim _{\tilde{u}_{0} \rightarrow 0}-\frac{\tilde{x}_{0}}{\tilde{u}_{0} \tilde{v}_{0}}=-\frac{1}{\tilde{v}_{0}} \frac{d \tilde{x}_{0}}{d \tilde{u}_{0}}(0) .
$$

Thus, $X_{0}$ evaluated at $U_{0}=0$ (which implies that $\tilde{u}_{0}=0$ ) indicates the direction of approach to the line $\tilde{x}_{0}=\tilde{u}_{0}=0$, and so opens out this region so that more detail can be seen.

With $\alpha=0.5$, the condition (43) for the ball to be thrown towards the wall before the initial bounce becomes

$$
\Omega_{0}>\frac{U_{0}}{6}
$$

and again, the dividing plane defined by $\Omega_{0}=U_{0} / 6$ is very close to the plane $\Omega_{0}=0$ on the plots shown in Fig. 6.

\section{The Plane $X=0$}

To study the nonlinear map defined by (38)-(40) analytically would be very difficult. However, we can gain more understanding of the behaviour of the map and of the regions $R_{m}$ by considering the dynamics on the two boundary planes of $W$ defined by $X=0$ and $U=0$. In this section, we consider the boundary plane $X=0$. The plane $U=0$ is studied in the next section.

One of the conditions in (42) that define $W$ is $X_{n}>0$. However, it is interesting to consider the boundary to this region defined by $X_{n}=0$ since this will help us to understand the dynamics for small positive values of $X_{n}$. From $(32), X_{n}=0$ requires that $\tilde{x}_{n}=0$. In terms of the behaviour of the ball, this implies that the ball bounces at the point where the wall and the floor meet. While our modelling has ignored this specific point, it is useful to consider the dynamics in this plane from a mathematical perspective as the limiting case of nearby points which are in $W$. 


\subsection{Analysis of the plane $X=0$}

We first note from (37) that

$$
\tilde{F}\left(0, \Omega_{n}\right)=\left|2 c_{2} \Omega_{n}+c_{1}\right| .
$$

Substituting this into (33) gives

$$
\begin{aligned}
F_{1}\left(0, \Omega_{n}, U_{n}\right) & =-\beta U_{n}\left[\left(2 c_{2} \Omega_{n}+c_{1}\right)+\left|2 c_{2} \Omega_{n}+c_{1}\right|\right] \\
& = \begin{cases}0 & \text { if } 2 c_{2} \Omega_{n}+c_{1} \leq 0 \\
-2 \beta U_{n}\left(2 c_{2} \Omega_{n}+c_{1}\right) & \text { if } 2 c_{2} \Omega_{n}+c_{1}>0\end{cases}
\end{aligned}
$$

We note that the condition $2 c_{2} \Omega_{n}+c_{1} \leq 0$ is equivalent to

$$
\Omega_{n} \leq-\frac{c_{1}}{2 c_{2}}=\frac{2 \alpha-5}{2(1+\alpha)}
$$

since $c_{2}>0$.

We define a straight line in this region by

$$
\ell_{1}\left(\Omega_{n}, U_{n}\right) \equiv \beta c_{1} U_{n}+10 c_{2}^{2}+2 c_{2} c_{3} \Omega_{n}=0 .
$$

Note that $\ell_{1}\left(\Omega_{n}, U_{n}\right)=-F_{3}\left(0, \Omega_{n}, U_{n}\right)$. There are now five different cases to consider.

1. $\Omega_{n}<-c_{1} /\left(2 c_{2}\right), \ell_{1}\left(\Omega_{n}, U_{n}\right) \neq 0$.

In this case, $F_{1}\left(0, \Omega_{n}, U_{n}\right)=0, F_{3}\left(0, \Omega_{n}, U_{n}\right) \neq 0$ and $F_{4}\left(0, \Omega_{n}\right) \neq 0$ and so, from (38), we must have $X_{n+1}=0$ also. We note that if $\ell_{1}\left(\Omega_{n}, U_{n}\right)>0$, then $U_{n+1}<0$. This corresponds to region (a) of Fig. 7. Similarly, if $\ell_{1}\left(\Omega_{n}, U_{n}\right)<0$, then $U_{n+1}>0$, which violates the first condition of (42), and hence is of no further interest. This corresponds to region (b) of Fig. 7.

2. $\Omega_{n}>-c_{1} /\left(2 c_{2}\right), \quad \ell_{1}\left(\Omega_{n}, U_{n}\right) \neq 0$.

In this case, $F_{1}\left(0, \Omega_{n}, U_{n}\right)>0, F_{3}\left(0, \Omega_{n}, U_{n}\right) \neq 0$ and $F_{4}\left(0, \Omega_{n}\right)>0$ and so, from (38), $X_{n+1} \neq 0$.

If $\ell_{1}\left(\Omega_{n}, U_{n}\right)<0$, then $U_{n+1}>0$ and $X_{n+1}<0$ which is not in $W$. This corresponds to region (f) of Fig. 7. Conversely, if $\ell_{1}\left(\Omega_{n}, U_{n}\right)>0$, then $U_{n+1}<0$ and $X_{n+1}>0$. However, to be in $W$, we also require that $X_{n+1}<2$. The boundary of this region can be found by solving $X_{n+1}=2$. Assuming that $X_{n}=0$ and $\Omega_{n}>-c_{1} /\left(2 c_{2}\right)$ gives

$$
\begin{aligned}
X_{n+1} & =-\frac{F_{1}\left(0, \Omega_{n}, U_{n}\right)}{F_{3}\left(0, \Omega_{n}, U_{n}\right) F_{4}\left(0, \Omega_{n}\right)} \\
& =-\frac{2 \beta U_{n}\left(2 c_{2} \Omega_{n}+c_{1}\right)}{\left[\beta c_{1} U_{n}+10 c_{2}^{2}+2 c_{2} c_{3} \Omega_{n}\right]\left[\beta\left(2 c_{2} \Omega_{n}+c_{1}\right)\right]} \\
& =-\frac{2 U_{n}}{\beta c_{1} U_{n}+10 c_{2}^{2}+2 c_{2} c_{3} \Omega_{n}} .
\end{aligned}
$$

Thus, $X_{n+1}=2$ when

$$
\ell_{2}\left(\Omega_{n}, U_{n}\right) \equiv\left(1+\beta c_{1}\right) U_{n}+10 c_{2}^{2}+2 c_{2} c_{3} \Omega_{n}=0 .
$$

On this line, $U_{n}<0$ if and only if

$$
5 c_{2}+c_{3} \Omega_{n}>0
$$


since $\beta, c_{1}, c_{2}>0$. If $\alpha>2 / 5$, then $c_{3}<0$ and this condition becomes $\Omega_{n}<-5 c_{2} / c_{3}$. Conversely, if $\alpha<2 / 5$, then $c_{3}>0$ and so condition (47) is $\Omega_{n}>-5 c_{2} / c_{3}$. Since $-5 c_{2} / c_{3}<-c_{1} / 2 c_{2}$ for all $\alpha$ satisfying $0<\alpha<2 / 5$, then (47) is satisfied in the whole of this region. Clearly, (47) is also satisfied when $\alpha=2 / 5$.

We note from (45) that as $U_{n} \rightarrow 0$, then $X_{n+1} \rightarrow 0$ also. Thus, we conclude that $0<X_{n+1}<2$ provided that $U_{n}<0, \ell_{2}\left(\Omega_{n}, U_{n}\right)>0$ and these conditions imply that $\Omega_{n}<-\frac{5 c_{2}}{c_{3}}$ when $\alpha>\frac{2}{5}$. Observe also that $U_{n+1}<0$ in this region since $\ell_{1}\left(\Omega_{n}, U_{n}\right)>0$ and so the next iterate satisfies all the conditions (42). This area is given by regions (c) and (d) of Fig. 7. Within this region, it is possible to find another region ((d) of Fig. 7) where $0<X_{n+2}<2$ and $U_{n+2}<0$. This region is bounded by the line defined by $X_{n+2}=2$ and is a complicated function of $\Omega_{n}$ and $U_{n}$. It can be shown that it intersects the $\Omega_{n}$ axis at

$$
\Omega_{n}=\frac{5 c_{2}\left(1-c_{3}^{2}\right)}{\alpha \beta-c_{3}+c_{3}^{3}} .
$$

It can be verified that this intersection point is always greater than $-c_{1} /\left(2 c_{2}\right)$ for all $\alpha>2 / 5$ and so always occurs in the region under consideration for this range of $\alpha$. When $\alpha=0.5$ and $\beta=0.9$, the intersection point is $\Omega_{n}=4875 / 2383=2.0457$.

3. $\Omega_{n}<-c_{1} /\left(2 c_{2}\right), \quad \ell_{1}\left(\Omega_{n}, U_{n}\right)=0$.

On this line, which separates regions (a) and (b) of Fig. 7, we find that $F_{1}\left(0, \Omega_{n}, U_{n}\right)=$ $F_{3}\left(0, \Omega_{n}, U_{n}\right)=0$ and $F_{4}\left(0, \Omega_{n}, U_{n}\right) \neq 0$ and so $X_{n+1}$ is not defined. However, a limiting process with $X_{n} \rightarrow 0$ can be used to determine a value of $X_{n+1}$ in this case. We will consider this case in more detail in Section 6.2.

4. $\Omega_{n}>-c_{1} /\left(2 c_{2}\right), \quad \ell_{1}\left(\Omega_{n}, U_{n}\right)=0$.

On this line, which separates regions (e) and (f) of Fig. 7, we find that $F_{1}\left(0, \Omega_{n}, U_{n}\right) \neq 0$, $F_{3}\left(0, \Omega_{n}, U_{n}\right)=0$ and $F_{4}\left(0, \Omega_{n}\right) \neq 0$ and so $X_{n+1}$ is not defined in this case. As the line is approached, $X_{n+1}$ tends to either $\infty$ or $-\infty$.

5. $\Omega_{n}=-c_{1} /\left(2 c_{2}\right)$.

Along this line, $F_{1}\left(0, \Omega_{n}, U_{n}\right)=F_{4}\left(0, \Omega_{n}\right)=0$ and so $X_{n+1}$ is not defined. In the plane $X_{n}=0$, the line $\Omega_{n}=-\frac{c_{1}}{2 c_{2}}$ is a line of discontinuity for the evaluation of $X_{n+1}$ since

$$
\begin{array}{r}
X_{n+1} \rightarrow 0 \text { for } \Omega_{n} \nearrow-\frac{c_{1}}{2 c_{2}}, \\
X_{n+1} \rightarrow-\frac{2 U_{n}}{\beta c_{1} U_{n}+\alpha} \text { for } \Omega_{n} \searrow-\frac{c_{1}}{2 c_{2}},
\end{array}
$$

where we have used (45) and (19).

With $\alpha=0.5$ and $\beta=0.9$, the lines which define the different regions are given by

$$
\Omega_{n}=-\frac{4}{3}, \quad \ell_{1}\left(\Omega_{n}, U_{n}\right)=\frac{18}{35} U_{n}+\frac{45}{98}-\frac{3}{98} \Omega_{n}=0, \quad \ell_{2}\left(\Omega_{n}, U_{n}\right)=\frac{53}{35} U_{n}+\frac{45}{98}-\frac{3}{98} \Omega_{n}=0 .
$$

These results, summarized in Fig. 7, show that region (a) maps back onto this half-plane $(X=0, U<0)$, region (b) maps onto the other half of this plane $(X=0, U>0)$, which is outside of $W$, regions (c) and (d) map into $W$ and regions (e) and (f) do not map into $W$. Note that the boundary of regions (a), (c) and (d) can clearly be seen in Fig. 6(a) on the plane $X_{0}=0$. Moreover, the boundary of region (d) can also be see on this plane in Fig. $6(b)$. 


\subsection{Analysis of the limit in case 3}

We recall that case 3 listed above is the line defined by

$$
\ell_{1}\left(\Omega_{n}, U_{n}\right)=0, \quad \Omega_{n}<-\frac{c_{1}}{2 c_{2}}
$$

and that this is the line that divides regions (a) and (b) of Fig. 7. Solving the defining equation for $U_{n}$ gives the alternative representation of the line as

$$
U_{n}=-\frac{10 c_{2}^{2}+2 c_{2} c_{3} \Omega_{n}}{\beta c_{1}} .
$$

Consider first the range of values of $\Omega_{n}$ which ensure that $U_{n}<0$ along this line. At the end point of the line $\Omega_{n}=-c_{1} /\left(2 c_{2}\right)$, we note that $U_{n}=-\alpha /\left(\beta c_{1}\right)<0$ (using (19)). When $\alpha \geq 2 / 5$, then $c_{3} \leq 0$ and so the slope of the line (50) is positive and this implies that $U_{n}<0$ along the whole line. However, if $\alpha<2 / 5$, then the line (50) has a negative slope and in this case, $U_{n}<0$ requires that

$$
5 c_{2}+c_{3} \Omega_{n}>0
$$

We observe for future reference that the inequality (51) also holds along the line when $\alpha \geq 2 / 5$.

We have already noted that $X_{n+1}$ is not defined on this line but if we consider nearby points with $X_{n}>0$ and take the limit as $X_{n} \rightarrow 0$, then a limiting value can be obtained. Consider the surface defined by

$$
\ell_{1}\left(\Omega_{n}, U_{n}\right)=\beta c_{1} U_{n}+10 c_{2}^{2}+2 c_{2} c_{3} \Omega_{n}=k X_{n},
$$

for some constant $k$. Clearly this corresponds to the line (49) when $X_{n}=0$. Substituting for $U_{n}$ from (52), we find that on this surface

$$
\begin{aligned}
& F_{1}\left(X_{n}, \Omega_{n}, U_{n}\right)=-\frac{\left(k X_{n}-10 c_{2}^{2}-2 c_{2} c_{3} \Omega_{n}\right)\left[2 c_{2} \Omega_{n}+c_{1}\left(1-X_{n}\right)+\tilde{F}\left(X_{n}, \Omega_{n}\right)\right]}{c_{1}}, \\
& F_{2}\left(X_{n}, \Omega_{n}, U_{n}\right)=-\frac{\alpha}{c_{1}}\left(5 c_{2}+c_{3} \Omega_{n}\right)-\frac{5 c_{2}}{c_{1}}\left(c_{1} c_{3}-k\right) X_{n}, \\
& F_{3}\left(X_{n}, \Omega_{n}, U_{n}\right)=\left(10 c_{2}^{2}-k\right) X_{n} .
\end{aligned}
$$

We also note that

$$
\begin{aligned}
\tilde{F}\left(X_{n}, \Omega_{n}\right) & =\sqrt{\left(2 c_{2} \Omega_{n}+c_{1}\right)^{2}}\left(1-\frac{c_{1}\left(2 c_{2} \Omega_{n}+c_{1}\right)-1}{\left(2 c_{2} \Omega_{n}+c_{1}\right)^{2}} X_{n}+O\left(X_{n}^{2}\right)\right) \\
& =-\left(2 c_{2} \Omega_{n}+c_{1}\right)+\frac{c_{1}\left(2 c_{2} \Omega_{n}+c_{1}\right)-1}{2 c_{2} \Omega_{n}+c_{1}} X_{n}+O\left(X_{n}^{2}\right),
\end{aligned}
$$

since $2 c_{2} \Omega_{n}+c_{1}<0$. Substituting this expansion into (53) gives

$$
F_{1}\left(X_{n}, \Omega_{n}, U_{n}\right)=\frac{\left(k X_{n}-10 c_{2}^{2}-2 c_{2} c_{3} \Omega_{n}\right)\left[X_{n}+O\left(X_{n}^{2}\right)\right]}{c_{1}\left(2 c_{2} \Omega_{n}+c_{1}\right)} .
$$

The limiting values as $X_{n} \rightarrow 0$ are now found as

$$
\begin{aligned}
X_{n+1} & =-\frac{2 c_{2}\left(5 c_{2}+c_{3} \Omega_{n}\right)}{\beta c_{1}\left(2 c_{2} \Omega_{n}+c_{1}\right)^{2}\left(10 c_{2}^{2}+k\right)}, \\
\Omega_{n+1} & =\frac{\alpha\left(5 c_{2}+c_{3} \Omega_{n}\right)}{\beta c_{1}\left(2 c_{2} \Omega_{n}+c_{1}\right)}, \\
U_{n+1} & =0 .
\end{aligned}
$$


We note that $\Omega_{n+1}<0$ (using (49) and (51)) and that the next iterate is on another boundary of $W$ as $U_{n+1}=0$. This boundary will be considered in more detail in Section 7 .

We now consider conditions on $k$ for the next iterate to lie in $W$, for which we require that $U_{n+1}<0$ and $0<X_{n+1}<2$. Using (54), we see that $U_{n+1}<0$ for small $X_{n}>0$ provided that

$$
k>10 c_{2}^{2} \text {. }
$$

By (56) and (51) this condition also ensures that $X_{n+1}>0$ in the limit as $X_{n} \rightarrow 0$.

From (56), the condition on $k$ such that $X_{n+1}<2$ is given by

$$
k>\frac{c_{2}\left(5 c_{2}+c_{3} \Omega_{n}\right)}{\beta c_{1}\left(2 c_{2} \Omega_{n}+c_{1}\right)^{2}}+10 c_{2}^{2} .
$$

The first term on the right hand side is positive (using (51)), and so if this inequality is satisfied, then so is (57). Thus, we conclude that all the required conditions hold if (58) is satisfied.

Note that it is possible to solve for $k$ the full equation $X_{n+1}=2$, where $F_{1}$ and $F_{3}$ are given by (53) and (54). Substituting this solution back into (52) gives the surface defined by

$$
U_{n}=-\frac{4 c_{2}\left(5 c_{2}\left(1-X_{n}\right)+c_{3} \Omega_{n}\right) \tilde{F}\left(X_{n}, \Omega_{n}\right)}{2 c_{2} \Omega_{n}+c_{1}\left(1-X_{n}\right)+\left(1+2 \beta c_{1}\right) \tilde{F}\left(X_{n}, \Omega_{n}\right)},
$$

which is shown in Fig. 8. Note how this surface intersects the plane $X_{n}=0$ along the lines which bound regions (a) and (c) of Fig. 7. The region above the plane (59) and bounded by the planes $U_{n}=0$ and the $X_{n}=0$ is the region $R_{2}$ which is shown in Fig. 6(a).

\subsection{Fixed point}

In the plane $X=0$, there is a fixed point $(X, \Omega, U)=\left(0, \Omega_{0}, U_{0}\right)$ in region (a) of Fig. 7 which can be found by solving the two equations

$$
\begin{aligned}
& \Omega_{0}=\frac{F_{2}\left(0, \Omega_{0}, U_{0}\right)}{F_{4}\left(0, \Omega_{0}\right)}=-\frac{5 \beta c_{2} U_{0}+5 c_{2} c_{3}+c_{3}^{2} \Omega_{0}}{\beta\left(2 c_{2} \Omega_{0}+c_{1}\right)}, \\
& U_{0}=\frac{F_{3}\left(0, \Omega_{0}, U_{0}\right)}{F_{4}\left(0, \Omega_{0}\right)}=\frac{\beta c_{1} U_{0}+10 c_{2}^{2}+2 c_{2} c_{3} \Omega_{0}}{\beta\left(2 c_{2} \Omega_{0}+c_{1}\right)},
\end{aligned}
$$

where we have used (55) to evaluate $F_{4}\left(0, \Omega_{0}\right)$. Solving $(60)$ for $U_{0}$ gives

$$
U_{0}=-\frac{\beta \Omega_{0}\left(2 c_{2} \Omega_{0}+c_{1}\right)+c_{3}\left(5 c_{2}+c_{3} \Omega_{0}\right)}{5 \beta c_{2}},
$$

and substituting into (61) provides the cubic equation

$$
2 \beta c_{2} \Omega_{0}^{3}+\left(\beta c_{1}+c_{3}^{2}\right) \Omega_{0}^{2}+10 c_{2} c_{3} \Omega_{0}+25 c_{2}^{2}=0 .
$$

Similarly, solving (61) for $\Omega_{0}$ and substituting into (60) gives another cubic equation

$$
\beta^{2} U_{0}^{3}-\beta c_{3} U_{0}^{2}+\beta c_{1} U_{0}+\alpha=0 .
$$

To investigate the character of the roots further, we form the discriminant $\Delta$, the product of the squared differences of the roots, of the cubic equation (63). We find that, in terms of $\alpha$ and $\beta$,

$\Delta=4900(1+\alpha)^{2}\left\{7(2 \alpha-5)^{3} \beta^{2}+\left(25 \alpha^{4}-2350 \alpha^{3}-9522 \alpha^{2}-2350 \alpha+25\right) \beta-7 \alpha(5 \alpha-2)^{3}\right\}$. 
If $\Delta<0$, the cubic (63) has one real and two complex conjugate roots, whereas if $\Delta \geq 0$, (63) has three real roots with a repeated root in the case of equality. Suppose now that we plot the curve $\Delta=0$ for $0<\alpha<1,0<\beta<1$. The result is a concave arc joining the points $(2 / 5,0)$ and $(0,1 / 35)$ (put $\beta=0$ and $\alpha=0$ in $\Delta=0$ ). Three negative real roots corresponds to values $(\alpha, \beta)$ in the area bounded by the arc and the segments $0<\alpha<2 / 5$ and $0<\beta<1 / 35$ of the axes. The remainder of the square $0<\alpha, \beta<1$ corresponds to values of $(\alpha, \beta)$ for which (63) has a single real root. The tiny $\beta$-values for which there are three real roots are of no interest physically and so we consider only the region where there is a single real root. Note that the same discriminant (up to a positive scalar) is obtained from the cubic equation (64).

We now show that the one real solution of the equations (60) and (61) lies in region (a) of Fig. 7. To do this, we must verify the three conditions (i) $U_{0}<0$, (ii) $2 c_{2} \Omega_{0}+c_{1}<0$ and (iii) $\ell_{1}\left(\Omega_{0}, U_{0}\right)>0$. We consider each of these conditions in turn.

(i) If $\alpha>2 / 5$, then all the coefficients in equation (64) are positive. Since there are no changes of sign in these coefficients, Descartes' rule of signs (see, for example, [13]) says that there are no positive roots to this equation, and either one or three negative real roots. Since we have a single root, it must be negative in this case.

If $\alpha<2 / 5$, then the coefficient of $U_{0}^{2}$ is negative, and so there are two changes of sign in the coefficients. In this case, Descartes' rule of signs implies that there are either two or zero positive real roots of the equation. Since we have only a single real root, this must correspond to the case of zero positive real roots and hence the one real root must be negative.

Thus, in both cases, the only real root of the equation must be negative and so $U_{0}<0$.

(ii) The cubic equation (63) can also be expressed as

$$
\left(5 c_{2}+c_{3} \Omega_{0}\right)^{2}=-\beta \Omega_{0}^{2}\left(2 c_{2} \Omega_{0}+c_{1}\right) .
$$

From this, it follows that any real solutions of this equation must satisfy $2 c_{2} \Omega_{0}+c_{1} \leq 0$. We note that equality is only possible if $5 c_{2}+c_{3} \Omega_{0}=2 c_{2} \Omega_{0}+c_{1}=0$ and it can be shown that this only holds if $\alpha=0$, which is outside our range of interest. Thus, we conclude that the real solution must satisfy $2 c_{2} \Omega_{0}+c_{1}<0$.

(iii) Solving (61) for $U_{0}$ gives

$$
U_{0}=\frac{5 c_{2}+c_{3} \Omega_{0}}{\beta \Omega_{0}}
$$

and substituting this value into $\ell_{1}\left(\Omega_{0}, U_{0}\right)$ gives

$$
\ell_{1}\left(\Omega_{0}, U_{0}\right)=\frac{\left(2 c_{2} \Omega_{0}+c_{1}\right)\left(5 c_{2}+c_{3} \Omega_{0}\right)}{\Omega_{0}}
$$

Now from (ii) we know that $2 c_{2} \Omega_{0}+c_{1}<0$ and we have also established that $\Omega_{0}<0$. Thus, it remains to determine the sign of $5 c_{2}+c_{3} \Omega_{0}$.

In (66), $U_{0}<0$ and $\Omega_{0}<0$ which imply that $5 c_{2}+c_{3} \Omega_{0}>0$. Thus, we conclude that $\ell_{1}\left(\Omega_{0}, U_{0}\right)>0$ as required. 
When $\alpha=0.5$ and $\beta=0.9$, the fixed point, as shown in Fig. 7 , is given by

$$
\Omega_{0}=-2.164886, \quad U_{0}=-0.629267 \text {. }
$$

We note that there is no corresponding fixed point in terms of the original non-dimensional variables $\tilde{x}, \tilde{\omega}, \tilde{u}$ and $\tilde{v}$. Having this fixed point and being able to analyse it is another advantage of studying the three-dimensional system in the scale-free variables.

Having determined that there is a fixed point in region (a) of Fig. 7, we now linearize about this fixed point and consider the eigenvalues of the Jacobian matrix evaluated at this point. The Jacobian is given by

$$
J\left(\Omega_{0}, U_{0}\right)=\left[\begin{array}{ccc}
j_{11}\left(\Omega_{0}, U_{0}\right) & 0 & 0 \\
j_{21}\left(\Omega_{0}, U_{0}\right) & j_{22}\left(\Omega_{0}, U_{0}\right) & j_{23}\left(\Omega_{0}, U_{0}\right) \\
j_{31}\left(\Omega_{0}, U_{0}\right) & j_{32}\left(\Omega_{0}, U_{0}\right) & j_{33}\left(\Omega_{0}, U_{0}\right)
\end{array}\right],
$$

where

$$
\begin{aligned}
j_{11}\left(\Omega_{0}, U_{0}\right) & =-\frac{U_{0}}{\left(\beta c_{1} U_{0}+10 c_{2}^{2}+2 c_{2} c_{3} \Omega_{0}\right)\left(2 c_{2} \Omega_{0}+c_{1}\right)^{2}}, \\
j_{21}\left(\Omega_{0}, U_{0}\right) & =-\frac{\left(2 c_{2} \Omega_{0}+c_{1}\right)\left(5 \beta c_{1} c_{2} U_{0}-\alpha c_{3} \Omega_{0}\right)-c_{3}\left(5 c_{2}+c_{3} \Omega_{0}\right)-5 \beta c_{2} U_{0}}{\beta\left(2 c_{2} \Omega_{0}+c_{1}\right)^{3}}, \\
j_{22}\left(\Omega_{0}, U_{0}\right) & =\frac{10 \beta c_{2}^{2} U_{0}+\alpha c_{3}}{\beta\left(2 c_{2} \Omega_{0}+c_{1}\right)^{2}}, \\
j_{23}\left(\Omega_{0}, U_{0}\right) & =-\frac{5 c_{2}}{2 c_{2} \Omega_{0}+c_{1}}, \\
j_{31}\left(\Omega_{0}, U_{0}\right) & =-\frac{\left(2 c_{2} \Omega_{0}+c_{1}\right)\left(2 \alpha c_{2} \Omega_{0}-\beta c_{1}^{2} U_{0}\right)+2 c_{2}\left(5 c_{2}+c_{3} \Omega_{0}\right)+\beta c_{1} U_{0}}{\beta\left(2 c_{2} \Omega_{0}+c_{1}\right)^{3}}, \\
j_{32}\left(\Omega_{0}, U_{0}\right) & =-\frac{2 c_{2}\left(\beta c_{1} U_{0}+\alpha\right)}{\beta\left(2 c_{2} \Omega_{0}+c_{1}\right)^{2}}, \\
j_{33}\left(\Omega_{0}, U_{0}\right) & =\frac{c_{1}}{2 c_{2} \Omega_{0}+c_{1}},
\end{aligned}
$$

again using (19). Clearly, the eigenvalues of $J\left(\Omega_{0}, U_{0}\right)$ are given by $\lambda_{1}=j_{11}\left(\Omega_{0}, U_{0}\right)$ together with the two eigenvalues $\lambda_{2,3}$ of the matrix

$$
\tilde{J}\left(\Omega_{0}, U_{0}\right)=\left[\begin{array}{cc}
j_{22}\left(\Omega_{0}, U_{0}\right) & j_{23}\left(\Omega_{0}, U_{0}\right) \\
j_{32}\left(\Omega_{0}, U_{0}\right) & j_{33}\left(\Omega_{0}, U_{0}\right)
\end{array}\right] .
$$

To find these two eigenvalues, we note that

$$
\operatorname{tr}\left(\tilde{J}\left(\Omega_{0}, U_{0}\right)\right)=\frac{10 \beta c_{2}^{2} U_{0}+\alpha c_{3}+\beta c_{1}\left(2 c_{2} \Omega_{0}+c_{1}\right)}{\beta\left(2 c_{2} \Omega_{0}+c_{1}\right)^{2}} .
$$

Substituting for $U_{0}$ from (62) gives

$$
\operatorname{tr}\left(\tilde{J}\left(\Omega_{0}, U_{0}\right)\right)=-\frac{\beta\left(2 c_{2} \Omega_{0}-c_{1}\right)+c_{3}^{2}}{\beta\left(2 c_{2} \Omega_{0}+c_{1}\right)} .
$$

Similarly,

$$
\operatorname{det}\left(\tilde{J}\left(\Omega_{0}, U_{0}\right)\right)=-\frac{\alpha^{2}}{\beta\left(2 c_{2} \Omega_{0}+c_{1}\right)^{3}},
$$


which is clearly positive since $2 c_{2} \Omega_{0}+c_{1}<0$ (see (ii) above).

When $\alpha=0.5$ and $\beta=0.9$, it is found that

$$
\operatorname{tr}\left(\tilde{J}\left(\Omega_{0}, U_{0}\right)\right)=-4.190944, \quad \operatorname{det}\left(\tilde{J}\left(\Omega_{0}, U_{0}\right)\right)=6.137029,
$$

and so the two eigenvalues in this particular case are given by $\lambda_{2,3}=-2.095472 \pm 1.321372 i$. We now show that these eigenvalues are complex for most values of $\alpha$ and $\beta$. To do this, we must show that the discriminant of the quadratic eigenvalue equation is negative. The boundary of this region occurs where the discriminant is zero, which is defined by the equation

$$
\begin{aligned}
& \operatorname{tr}\left(\tilde{J}\left(\Omega_{0}, U_{0}\right)\right)^{2}-4 \operatorname{det}\left(\tilde{J}\left(\Omega_{0}, U_{0}\right)\right)= \\
& \quad \frac{8 \beta^{2} c_{2}^{3} \Omega_{0}^{3}+4 \beta c_{2}^{2}\left(2 c_{3}^{2}-\beta c_{1}\right) \Omega_{0}^{2}+2 c_{2}\left(c_{3}^{4}-\beta^{2} c_{1}^{2}\right) \Omega_{0}+c_{1}\left(\beta c_{1}-c_{3}^{2}\right)^{2}+4 \alpha^{2} \beta}{\beta^{2}\left(2 c_{2} \Omega_{0}+c_{1}\right)^{3}}=0 .
\end{aligned}
$$

The problem we have now is that $\Omega_{0}$ is the solution of the cubic equation (63) and so is not easily obtained in a form that can be substituted into this equation. Thus, we regard (63) as an equation involving $\Omega_{0}, \alpha$ and $\beta$ and solve it for $\beta$. Substituting this value of $\beta$ into (69) gives an equation which involves only $\Omega_{0}$ and $\alpha$, and which is quadratic in $\Omega_{0}$. This equation can be solved for $\Omega_{0}$ in terms of $\alpha$. Substituting these solutions back into (63) gives an equation involving only $\alpha$ and $\beta$ and which can be solved for $\beta$. One of the two solutions gives rise to negative values of $\beta$ and so is of no interest. The other gives a complicated function of $\alpha$ which is plotted as the blue line in Fig. 9. It is easily verified that the eigenvalues are complex in the large region above this line, and real in the small region below it. Clearly this small region is of no interest to us as $\beta$ is very small there.

Consider next the magnitude of $\left|\lambda_{2,3}\right|$, since this determines whether the fixed point is attracting or repelling in the plane. We note that

$$
\operatorname{det} \tilde{J}\left(\Omega_{0}, U_{0}\right)=\left|\lambda_{2,3}\right|^{2}
$$

and so the boundary between stability and instability of the fixed point (in the plane) is defined by

$$
\operatorname{det} \tilde{J}\left(\Omega_{0}, U_{0}\right)=1 \text {. }
$$

Solving (63) in the factorised form (65) for $\beta$ and substituting into (68) gives

$$
\operatorname{det} \tilde{J}\left(\Omega_{0}, U_{0}\right)=\left(\frac{\alpha \Omega_{0}}{\left(5 c_{2}+c_{3} \Omega_{0}\right)\left(2 c_{2} \Omega_{0}+c_{1}\right)}\right)^{2},
$$

and so (70) is equivalent to

$$
\left(5 c_{2}+c_{3} \Omega_{0}\right)\left(2 c_{2} \Omega_{0}+c_{1}\right)= \pm \alpha \Omega_{0}
$$

For $\alpha \geq 2 / 5, c_{3} \leq 0$ and so $5 c_{2}+c_{3} \Omega_{0}>0$. Since $2 c_{2} \Omega_{0}+c_{1}<0$, we must take the positive sign in the above equation in this case. Solving for $\Omega_{0}$ in terms of $\alpha$, we find that

$$
2 c_{2} \Omega_{0}+c_{1}=-\sqrt{-\frac{\alpha c_{1}}{c_{3}}}
$$


where we have taken the negative square root since $2 c_{2} \Omega_{0}+c_{1}<0$. Solving (70) with the determinant given by (68) for $\beta$ and substituting using (72) gives

$$
\begin{aligned}
\beta & =-\frac{\alpha^{2}}{\left(2 c_{2} \Omega_{0}+c_{1}\right)^{3}} \\
& =\sqrt{\alpha}\left(-\frac{c_{3}}{c_{1}}\right)^{3 / 2} \\
& =\sqrt{\alpha}\left(\frac{5 \alpha-2}{5-2 \alpha}\right)^{3 / 2}
\end{aligned}
$$

This curve is plotted as the solid red line in Fig. 9. There are four solutions in total of (71), of which we have considered only one. For the other three, two have $\beta$ negative and the other curve lies in the region where the eigenvalues are real, and hence is of no interest.

Finally, we consider the eigenvalue $\lambda_{1}=j_{11}\left(\Omega_{0}, U_{0}\right)$. Substituting using $(61)$ in $j_{11}\left(\Omega_{0}, U_{0}\right)$ gives

$$
\lambda_{1}=-\frac{1}{\beta\left(2 c_{2} \Omega_{0}+c_{1}\right)^{3}} .
$$

Clearly, $\lambda_{1}>0$ since $2 c_{2} \Omega_{0}+c_{1}<0$. When $\alpha=0.5$ and $\beta=0.9$, we find that $\lambda_{1}=24.5481$ with corresponding eigenvector $v_{1}$ given by

$$
v_{1}=\left[\begin{array}{c}
1 \\
0.7940 \\
0.2627
\end{array}\right] \text {. }
$$

This eigenvector gives the tangent direction to the one-dimensional unstable manifold from the fixed point out of the plane $X=0$. This unstable manifold is easy to find by iterating a small line segment from the fixed point in the direction of the eigenvector. Since the eigenvalue $\lambda_{1}$ is so large, the iterates very quickly escape from $W$ along this manifold. Two projections of the manifold are shown in Fig. 10 together with three iterates on the manifold.

Stability of the fixed point with respect to perturbations out of the plane is determined by the magnitude of $\lambda_{1}$. In particular, the boundary between stability and instability is defined by $\lambda_{1}=1$. We note that this condition is very similar to the condition $\operatorname{det}\left(\tilde{J}\left(\Omega_{0}, U_{0}\right)\right)=1$, where $\operatorname{det}\left(\tilde{J}\left(\Omega_{0}, U_{0}\right)\right)$ is given by $(68)$. Thus, we use a similar approach to determine the line in parameter space corresponding to $\lambda_{1}=1$. It can be shown that this line is given by

$$
\beta=-\left(\frac{c_{3}}{c_{1}}\right)^{3}
$$

and this is illustrated by the dashed line in Fig. 9. It is easily verified that $\lambda_{1}>1$ to the left of this line and that $\lambda_{1}<1$ to the right of the line.

If a material could be found which gave coefficients of restitution $\alpha$ and $\beta$ in region (c) of Fig. 9, then this would imply that a large region of the space of initial conditions would give rise to trajectories that converge to the fixed point and thus do not escape from $W$. Physically, this implies that there are infinitely many bounces of the ball between the floor and the wall. Simulations with $\alpha=0.95, \beta=0.6$ confirm these conclusions. One such trajectory of the ball is shown in Fig. 11.

We summarize all these results as follows. 


\section{Theorem 6.1}

For all values of $\beta$ satisfying $1 / 35<\beta<1$, the plane $X=0$ has a fixed point in region (a) of Fig. 7. The two eigenvalues of the Jacobian evaluated at the fixed point associated with perturbations in the plane are always complex, so that iterates near to the fixed point spiral around it. The eigenvalue associated with perturbations out of the plane is positive. As $\alpha$ increases from zero to one for a fixed value of $\beta$, three scenarios are encountered which are, in order,

(i) all three eigenvalues are unstable, and so the fixed point is linearly unstable with respect to all perturbations (region (a) in Fig. 9);

(ii) the two complex eigenvalues are stable while the real eigenvalue is unstable. Thus, iterates in the plane sufficiently close to the fixed point will converge to the fixed point, while iterates near the fixed point but out of the plane will spiral around the one-dimensional unstable manifold of the fixed point, moving away from the plane $X=0$ but converging towards the unstable manifold (region (b) in Fig. 9);

(iii) all three eigenvalues are stable, and so the fixed point is linearly stable with respect to all perturbations (region (c) in Fig. 9).

\subsection{Iteration in region (a) of Fig. 7}

We recall that iterates in region (a) of Fig. 7 map to somewhere else in the half-plane defined by $X_{n}=0, U_{n}<0$ and so some parts of region (a) will map back onto region (a). However, the regions for which this holds will clearly get smaller for more iterations, assuming that the parameters $\alpha$ and $\beta$ are chosen in region (a) of Fig. 9, so that the fixed point is linearly unstable in the plane. To determine these regions, the boundary lines of region (a) defined by $\Omega_{n}=-c_{1} /\left(2 c_{2}\right), \ell_{1}\left(\Omega_{n}, U_{n}\right)=0$ and $U_{n}=0$ can be back iterated.

The iteration on the part of the plane defined by $\Omega_{n}<-c_{1} /\left(2 c_{2}\right)$ is given by

$$
\begin{aligned}
\Omega_{n+1} & =-\frac{5 \beta c_{2} U_{n}+5 c_{2} c_{3}+c_{3}^{2} \Omega_{n}}{\beta\left(2 c_{2} \Omega_{n}+c_{1}\right)}, \\
U_{n+1} & =\frac{\beta c_{1} U_{n}+10 c_{2}^{2}+2 c_{2} c_{3} \Omega_{n}}{\beta\left(2 c_{2} \Omega_{n}+c_{1}\right)}
\end{aligned}
$$

since $\tilde{F}\left(0, \Omega_{n}\right)=\left|2 c_{2} \Omega_{n}+c_{1}\right|=-\left(2 c_{2} \Omega_{n}+c_{1}\right)$. These equations can be solved for $\Omega_{n}$ and $U_{n}$ to give the inverse map explicitly, but we do not require this form.

We can now back iterate each of the three boundary lines. We start by setting $\Omega_{n+1}=$ $-c_{1} /\left(2 c_{2}\right)$ and substituting this into (73) gives the line

$$
U_{n}=\frac{2 c_{2}\left(\beta c_{1}-c_{3}^{2}\right) \Omega_{n}+\beta c_{1}^{2}-10 c_{2}^{2} c_{3}}{10 \beta c_{2}^{2}} .
$$

Similarly, we write the second line as $\ell_{1}\left(\Omega_{n+1}, U_{n+1}\right)=0$ and substituting for $\Omega_{n+1}$ and $U_{n+1}$ from (73) and (74) gives

$$
U_{n}=-\frac{2 c_{2}}{\beta\left(\beta c_{1}^{2}-10 c_{2}^{2} c_{3}\right)}\left[\left(10 \beta c_{2}^{2}+\beta c_{1} c_{3}-c_{3}^{3}\right) \Omega_{n}+10 \beta c_{1} c_{2}-5 c_{2} c_{3}^{2}\right]
$$


The third boundary line is $U_{n+1}=0$ and substituting this into (74), we derive the preimage

$$
U_{n}=-\frac{10 c_{2}^{2}+2 c_{2} c_{3} \Omega_{n}}{\beta c_{1}},
$$

which we note is the line $\ell_{1}\left(\Omega_{n}, U_{n}\right)=0$.

The region defined by these three preimage lines must be partly cut off by the line $\Omega_{n}=-c_{1} /\left(2 c_{2}\right)$, so that the resulting region is bounded by a quadrilateral. For $\alpha=0.5$ and $\beta=0.9$ the required region is shown in Fig. 12 as the lightest shaded area, which is the preimage of region (a). Further inverse iteration of this quadrilateral region can be performed, resulting in smaller regions at each stage which are converging towards the fixed point found previously in Section 6.3. The next few steps of this process are also shown in Fig. 12.

Clearly some points in region (a) will end up, under forward iteration, in regions (c), (d) or (e) of Fig. 7. Thus, we can define more regions of this plane where $0<X_{n+k}<2$ and $U_{n+k}<0$ for increasing values of $k$.

We first note that for $\alpha=0.5, \beta=0.9$, if $\left(\Omega_{n}, U_{n}\right)$ is in region (a), then $\left(\Omega_{n+1}, U_{n+1}\right)$ will lie to the left of the line $U_{n+1}=6 \Omega_{n+1}$, which is the forward iterate of the line $U_{n}=$ 0 . In particular, this implies that $\Omega_{n+1}$ is always negative. Thus, for these parameter values, iterates of points in region (a) will never lie in region (d). It is likely that this will also be true for many other parameter values, but we have not considered this in detail. However, we concentrate our attention on region (c), since this is the region for which $0<X_{n+1}<2$ and $U_{n+1}<0$. It is bounded by the lines $U_{n}=0, \Omega_{n}=-c_{1} /\left(2 c_{2}\right)$ and $U_{n}=-2 c_{2}\left(5 c_{2}+c_{3} \Omega_{n}\right) /\left(1+\beta c_{1}\right)$. We have already back iterated the first two of these lines, and so it remains to find the inverse iterate of the third. Using a similar process to before, this gives the line

$$
U_{n}=-\frac{2 c_{2}}{\beta\left(\beta c_{1}^{2}+c_{1}-10 c_{2}^{2} c_{3}\right)}\left[\left(10 \beta c_{2}^{2}+\beta c_{1} c_{3}+c_{3}-c_{3}^{3}\right) \Omega_{n}+10 \beta c_{1} c_{2}+5 c_{2}-5 c_{2} c_{3}^{2}\right] .
$$

For future reference, we note that this line intersects the $\Omega_{n}$-axis at

$$
\Omega_{n}=-\frac{10 \beta c_{1} c_{2}+5 c_{2}-5 c_{2} c_{3}^{2}}{10 \beta c_{2}^{2}+\beta c_{1} c_{3}+c_{3}-c_{3}^{3}} .
$$

Thus, the region for which $0<X_{n+2}<2$ and $U_{n+2}<0$ is bounded by the lines (75), (76) and (77) and is shown as the lightest shaded red region in Fig. 13. Back iterating this region gives the next darkest shaded region, which is now also cut off by the lines $\Omega_{n}=-c_{1} /\left(2 c_{2}\right)$ and $\ell_{1}\left(\Omega_{n}, U_{n}\right)=0$, resulting in a pentagonal region. This region can be further back iterated as shown in Fig. 13, again resulting in smaller regions at each stage and converging towards the fixed point.

Combining these two sets of results, we can find regions of the plane $X_{n}=0$ where $0 \leq X_{n+k}<2$ (note the equality!) and $U_{n+k}<0$. These regions consist of the combination of the corresponding blue and red areas from Figs. 12 and 13 and are shown in Fig. 14.

Taking $n=0$, clearly these regions of the plane $X=0$ show where the regions $R_{m}$, $m=3,4, \ldots$ intersect this boundary plane. In Fig. $6(b)$, the intersection with the plane $X_{0}=0$ is similar to the region bounded by the blue line of Fig. 14. If the resolution used to generate Fig. $6(b)$ was finer, then better agreement would be obtained. Similarly the regions where $R_{4}$ and $R_{5}$ intersect the plane $X_{0}$ in Fig. 6(c), $(d)$ should match with the regions bounded by the red and green lines of Fig. 14, but again the resolution in Fig. 6 is poor. 


\subsection{The iteration for $X_{n}>0$}

We have so far studied in detail the dynamics in the plane $X=0$, even though this is only the boundary of the region $W$ but is not in $W$. However, the dynamics near to this boundary can be understood in terms of the dynamics on this boundary plane.

Let $\left(X_{n}, \Omega_{n}, U_{n}\right)$ be a point at which the functions $G_{1}, G_{2}$ and $G_{3}$ defined by (38)-(40) are differentiable. Then taking a Taylor series of these functions in the variable $X_{n}$ about $X_{n}=0$ gives

$$
\begin{aligned}
& X_{n+1}=G_{1}\left(X_{n}, \Omega_{n}, U_{n}\right)=G_{1}\left(0, \Omega_{n}, U_{n}\right)+G_{1, X}\left(0, \Omega_{n}, U_{n}\right) X_{n}+O\left(X_{n}^{2}\right), \\
& \Omega_{n+1}=G_{2}\left(X_{n}, \Omega_{n}, U_{n}\right)=G_{2}\left(0, \Omega_{n}, U_{n}\right)+G_{2, X}\left(0, \Omega_{n}, U_{n}\right) X_{n}+O\left(X_{n}^{2}\right), \\
& U_{n+1}=G_{3}\left(X_{n}, \Omega_{n}, U_{n}\right)=G_{3}\left(0, \Omega_{n}, U_{n}\right)+G_{3, X}\left(0, \Omega_{n}, U_{n}\right) X_{n}+O\left(X_{n}^{2}\right),
\end{aligned}
$$

where $G_{1, X}=\partial G_{1} / \partial X$, etc. Thus, the iterates are determined by the iterates in the plane $X_{n}=0$, perturbed by terms of $O\left(X_{n}\right)$, which we assume to be initially small. Moreover, if the point $\left(\Omega_{n}, U_{n}\right)$ is in region (a) of Fig. 7 , then $G_{1}\left(0, \Omega_{n}, U_{n}\right)=0$ and so

$$
X_{n+1}=G_{1, X}\left(0, \Omega_{n}, U_{n}\right) X_{n}+O\left(X_{n}^{2}\right) .
$$

We note that $G_{1, X}\left(0, \Omega_{n}, U_{n}\right)=j_{11}\left(\Omega_{n}, U_{n}\right)$, given by $(67)$. This function tends to $\infty$ for points that tend towards either of the boundary lines $\Omega_{n}=-c_{1} /\left(2 c_{2}\right)$ or $\ell_{1}\left(\Omega_{n}, U_{n}\right)=0$ and is zero along the remaining boundary line $U_{n}=0$. Solving the equation $j_{11}\left(\Omega_{n}, U_{n}\right)=1$ gives the line

$$
U_{n}=-\frac{2 c_{2}\left(2 c_{2} \Omega_{n}+c_{1}\right)^{2}\left(5 c_{2}+c_{3} \Omega_{n}\right)}{1+\beta c_{1}\left(2 c_{2} \Omega_{n}+c_{1}\right)^{2}},
$$

and this line is shown in Fig. 15 for $\alpha=0.5$ and $\beta=0.9$. Clearly, if $\left(\Omega_{n}, U_{n}\right)$ is a point below this line, then $X_{n+1}>X_{n}$ and if it is a point above the line, then $X_{n+1}<X_{n}$.

In general terms, if $\left(\Omega_{n}, U_{n}\right)$ is a point in region (a) of Fig. 7 and $X_{n}$ is small and positive, then the iterates will spiral out from the fixed point, while generally moving away monotonically from the $X=0$ plane (unless the dashed line in Fig. 15 is crossed) until an iterate leaves region (a), in which case the next iterate will either leave $W$ or jump away from the plane $X=0$ while remaining in $W$.

We note that $G_{1}\left(0, \Omega_{n}, U_{n}\right)$ is not defined along the line $\ell_{1}\left(\Omega_{n}, U_{n}\right)=0$, as considered in case 3 above and so the Taylor series approach cannot be used on this line. However, the behaviour of iterates with $X_{n}>0$ but near to this line was studied in Section 6.2.

\section{The Plane $U=0$}

The conditions (42) which define the region $W$ include $U_{n}<0$. As in the previous section, it is interesting to consider the boundary of $W$ defined by $U_{n}=0$, since this will help to understand the dynamics for small, negative values of $U_{n}$. From a physical point of view, this condition implies that the ball bounces vertically from the floor, which necessarily implies that it will not hit the wall before returning to the floor. However, insight can be gained by considering this plane from a mathematical perspective as the limiting case of nearby points which are in $W$.

We first consider which points $\left(X_{n}, \Omega_{n}\right)$ in the plane $U_{n}=0$ ensure that $U_{n+1}<0$. The boundary to this region will occur when $U_{n+1}=0$, and this occurs when

$$
F_{3}\left(X_{n}, \Omega_{n}, 0\right)=-10 c_{2}^{2}\left(1-X_{n}\right)-2 c_{2} c_{3} \Omega_{n}=0 .
$$


This is equivalent to

$$
X_{n}=L_{1}\left(\Omega_{n}\right) \equiv \frac{c_{3}}{5 c_{2}} \Omega_{n}+1 .
$$

It is easily verified that $U_{n+1}>0$ when $X_{n}>L_{1}\left(\Omega_{n}\right)$ and so this immediately implies that the region of interest in the plane $U_{n}=0$ is initially given by

$$
0<X_{n}<L_{1}\left(\Omega_{n}\right) .
$$

The behaviour of iterates near to the line $X_{n}=L_{1}\left(\Omega_{n}\right)$ with $U_{n} \neq 0$ is also of interest. We consider these two cases separately.

\subsection{The region $0<X_{n}<L_{1}\left(\Omega_{n}\right)$}

In the region defined by (79), $X_{n+1}=0$ which is one of the other boundary planes that we considered in the previous section. We note that in this case,

$$
\frac{\Omega_{n+1}}{U_{n+1}}=\frac{F_{2}\left(X_{n}, \Omega_{n}, 0\right)}{F_{3}\left(X_{n}, \Omega_{n}, 0\right)}=-\frac{c_{3}}{2 c_{2}},
$$

and so the region defined by (79) in the plane $U_{n}=0$ maps onto the line

$$
\Omega_{n+1}=-\frac{c_{3}}{2 c_{2}} U_{n+1}
$$

in the plane $X_{n+1}=0$. We note that points $\left(X_{n}, \Omega_{n}\right)$ in the plane $U_{n}=0$ that approach the line $X_{n}=L_{1}\left(\Omega_{n}\right)$ map, under iteration, to points on this line that approach the origin $U_{n+1}=\Omega_{n+1}=0$.

We now determine where the line (80) intersects the line $\ell_{2}\left(\Omega_{n+1}, U_{n+1}\right)=0$ which is defined by (46). This point of intersection is given by

$$
\left(\Omega_{n+1}^{(2)}, U_{n+1}^{(2)}\right)=\left(\frac{5 c_{2} c_{3}}{\beta c_{1}+1-c_{3}^{2}},-\frac{10 c_{2}^{2}}{\beta c_{1}+1-c_{3}^{2}}\right) .
$$

It can be shown that the function $\Omega_{n+1}^{(2)}+c_{1} /\left(2 c_{2}\right)$ is positive for all $\alpha, \beta \in[0,1]$, which implies that this point of intersection lies to the right of the line $\Omega_{n+1}=-c_{1} /\left(2 c_{2}\right)$. From this, we conclude that the line (80) intersects regions (c) and (e) of Fig. 7 for all $\alpha, \beta \in[0,1]$.

Similarly, the point of intersection of the line $\ell_{1}\left(\Omega_{n+1}, U_{n+1}\right)=0$ defined by (44) and the line (80) is found to be

$$
\left(\Omega_{n+1}^{(1)}, U_{n+1}^{(1)}\right)=\left(\frac{5 c_{2} c_{3}}{\beta c_{1}-c_{3}^{2}},-\frac{10 c_{2}^{2}}{\beta c_{1}-c_{3}^{2}}\right) .
$$

In this case, solving

$$
\Omega_{n+1}^{(1)}+\frac{c_{1}}{2 c_{2}}=0
$$

gives $\beta=-\alpha c_{3} / c_{1}^{2}$, using (19). To the left of this line in the parameter plane, the point of intersection $\left(\Omega_{n+1}^{(1)}, U_{n+1}^{(1)}\right)$ lies to the right of the line $\Omega_{n+1}=-c_{1} /\left(2 c_{2}\right)$, which implies that the line (80) intersects region (f) of Fig. 7. However, to the right of this line, the point of intersection $\left(\Omega_{n+1}^{(1)}, U_{n+1}^{(1)}\right)$ lies to the left of the line $\Omega_{n+1}=-c_{1} /\left(2 c_{2}\right)$, which implies that the line (80) intersects regions (a) and (b) of Fig. 7. In this case, it is possible that period 2 
orbits of the map may exist, since points that approach the line $\ell_{1}\left(\Omega_{n}, U_{n}\right)=0$ as $X_{n} \rightarrow 0$ map onto the plane $U_{n+1}=0$ (see Section 6.2) and some points on this plane map back onto the line (80) in the plane $X_{n+2}=0$ which intersects the line $\ell_{1}\left(\Omega_{n}, U_{n}\right)=0$.

The example that we are considering of $\alpha=0.5, \beta=0.9$ corresponds to the case where the line (80) intersects region (f), and so we do not consider the possibility of period two points further.

We now determine the line in the $U_{n}=0$ plane which maps onto the intersection point $\left(\Omega_{n+1}^{(2)}, U_{n+1}^{(2)}\right)$ in the plane $X_{n+1}=0$. This can be found by solving

$$
\begin{aligned}
\Omega_{n+1}^{(2)} & =\frac{F_{2}\left(X_{n}, \Omega_{n}, 0\right)}{F_{4}\left(X_{n}, \Omega_{n}, 0\right)} \\
& =\frac{5 c_{2} c_{3}\left(1-X_{n}\right)+c_{3}^{2} \Omega_{n}}{\beta \sqrt{\left[2 c_{2} \Omega_{n}+c_{1}\left(1-X_{n}\right)\right]^{2}+2 X_{n}-X_{n}^{2}}} .
\end{aligned}
$$

Substituting for $\Omega_{n+1}^{(2)}$, squaring and simplifying gives

$$
\left[\left(5 c_{2}\left(1-X_{n}\right)+c_{3} \Omega_{n}\right)\left(\beta c_{1}+1-c_{3}^{2}\right)\right]^{2}=\left(5 \beta c_{2}\right)^{2}\left[\left(2 c_{2} \Omega_{n}+c_{1}\left(1-X_{n}\right)\right)^{2}+2 X_{n}-2 X_{n}^{2}\right] .
$$

This equation is quadratic in $X_{n}$ (and in $\Omega_{n}$ ). We note that of the two solutions to this equation, only one of them will be a solution of the original equation as the second has been introduced due to squaring. The solutions are complicated functions of $\alpha$ and $\beta$. We denote the one solution of the original equation by $X_{n}=L_{2}\left(\Omega_{n}\right)$ and the graph of this solution for $\alpha=0.5, \beta=0.9$ is shown in Fig. 16. It is easily verified that the region between the lines $X_{n}=L_{1}\left(\Omega_{n}\right)$ and $X_{n}=L_{2}\left(\Omega_{n}\right)$ maps onto the intersection of the line (80) with region (c) of Fig. 7.

The two intersection points of the line $X_{n}=L_{2}\left(\Omega_{n}\right)$ with the $\Omega_{n}$-axis can be found and are given by (48) and (78). Thus, this line in the plane $U_{n}=0$ joins up with the boundary of region (d) of Fig. 7 in the plane $X_{n}=0$ and the boundary of the lightest red region of Fig. 13, again in the plane $X_{n}=0$.

For the parameter values $\alpha=0.5, \beta=0.9$, the line (80) does not intersect the boundary of region (d). (This is likely to be the case also for many other values of $\alpha$ and $\beta$, but we do not explore this in detail.) Thus, in this case, the region in the plane $U_{n}=0$ bounded by the lines $X_{n}=L_{1}\left(\Omega_{n}\right)$ and $X_{n}=L_{2}\left(\Omega_{n}\right)$ iterates as follows:

- in one iteration, it maps onto the intersection of the line (80) with region (c) of Fig. 7 in the boundary plane $X_{n+1}=0$;

- in two iterations it maps into $W$, so that $0<X_{n+2}<2$ and $U_{n+2}<0$;

- in three iterations, it escapes from $W$.

Thus, this region defines the boundary of the set $R_{3}$ (see Section 5 ) where it intersects the $U_{n}=0$ plane, as can be seen from the numerical results in Fig. 6(b).

When $U_{n}$ is small but non-zero, a similar approach to that used in Section 6.5 can be used to show that iterates are $O\left(U_{n}\right)$ from the corresponding iterates starting with $U_{n}=0$.

One question of interest in this case is whether $X_{n+1}>0$ for sufficiently small $U_{n}<0$ and $0<X_{n}<L_{1}\left(\Omega_{n}\right)$ ? We note from (38), (33) and (36) that

$$
X_{n+1}=\frac{U_{n}\left[2 c_{2} \Omega_{n}+c_{1}\left(1-X_{n}\right)+\tilde{F}\left(X_{n}, \Omega_{n}\right)\right]}{F_{3}\left(X_{n}, \Omega_{n}, U_{n}\right) \tilde{F}\left(X_{n}, \Omega_{n}\right)} .
$$


Now $\tilde{F}\left(X_{n}, \Omega_{n}\right)>0$ by definition. The region $0<X_{n}<L_{1}\left(\Omega_{n}\right)$ is defined to ensure that $F_{3}\left(X_{n}, \Omega_{n}, 0\right)<0$ and so, for sufficiently small $U_{n}, F_{3}\left(X_{n}, \Omega_{n}, U_{n}\right)<0$ also. Therefore, the denominator of (81) is negative. In the numerator, we have by assumption that $U_{n}<0$, and so it remains to consider the term in the square brackets. The following result shows that this quantity is positive.

\section{Lemma 7.1}

If $0<X_{n}<2$ then

$$
2 c_{2} \Omega_{n}+c_{1}\left(1-X_{n}\right)+\tilde{F}\left(X_{n}, \Omega_{n}\right)>0,
$$

where $\tilde{F}\left(X_{n}, \Omega_{n}\right)$ is defined by $(37)$.

\section{Proof}

We first define

$$
\hat{F}\left(X_{n}, \Omega_{n}\right)=2 c_{2} \Omega_{n}+c_{1}\left(1-X_{n}\right) .
$$

The expression of interest can then be rewritten as

$$
2 c_{2} \Omega_{n}+c_{1}\left(1-X_{n}\right)+\tilde{F}\left(X_{n}, \Omega_{n}\right)=\hat{F}\left(X_{n}, \Omega_{n}\right)+\sqrt{\hat{F}\left(X_{n}, \Omega_{n}\right)^{2}+2 X_{n}-X_{n}^{2}} .
$$

Since $0<X_{n}<2$, it follows that $2 X_{n}-X_{n}^{2}>0$ and so

$$
\hat{F}\left(X_{n}, \Omega_{n}\right)^{2}+2 X_{n}-X_{n}^{2}>\hat{F}\left(X_{n}, \Omega_{n}\right)^{2} \geq 0 .
$$

The square-root function is monotonically increasing, and so we conclude that

$$
\sqrt{\hat{F}\left(X_{n}, \Omega_{n}\right)^{2}+2 X_{n}-X_{n}^{2}}>\left|\hat{F}\left(X_{n}, \Omega_{n}\right)\right| \text {. }
$$

Hence,

$$
\begin{aligned}
\hat{F}\left(X_{n}, \Omega_{n}\right)+\sqrt{\hat{F}\left(X_{n}, \Omega_{n}\right)^{2}+2 X_{n}-X_{n}^{2}} & >\hat{F}\left(X_{n}, \Omega_{n}\right)+\left|\hat{F}\left(X_{n}, \Omega_{n}\right)\right| \\
& \geq 0
\end{aligned}
$$

which gives the required result.

Combining the signs of all these terms, we conclude that $X_{n+1}>0$, and so the first iterate is on the valid side of the boundary plane $X_{n+1}=0$. We finally note that this also implies that the region of the plane $U_{n}=0$ defined by $0<X_{n}<L_{1}\left(\Omega_{n}\right)$ must define the intersection of the set $R_{2}$ with the plane $U_{n}=0$. This region can clearly be seen in Fig. 6(a).

\subsection{The line $X_{n}=L_{1}\left(\Omega_{n}\right)$}

The line $X_{n}=L_{1}\left(\Omega_{n}\right)$ is found from the condition that $F_{3}\left(X_{n}, \Omega_{n}, 0\right)=0$ and so, from (38), $X_{n+1}$ is not defined along this line. However, a limiting process with $U_{n} \rightarrow 0$ can be used to determine a value of $X_{n+1}$ in this case, using a similar approach to that used in Section 6.2.

We consider the surface defined by

$$
-10 c_{2}^{2}\left(1-X_{n}\right)-2 c_{2} c_{3} \Omega_{n}=k U_{n}
$$


for some $k$. Clearly, this corresponds to the line $X_{n}=L_{1}\left(\Omega_{n}\right)$ when $U_{n}=0$. On this surface, we find that

$$
\begin{aligned}
& F_{2}\left(X_{n}, \Omega_{n}, U_{n}\right)=\left(5 \beta c_{2}-\frac{k c_{3}}{2 c_{2}}\right) U_{n} \\
& F_{3}\left(X_{n}, \Omega_{n}, U_{n}\right)=\left(k-\beta c_{1}\right) U_{n}
\end{aligned}
$$

Clearly, $F_{4}\left(X_{n}, \Omega_{n}\right)$ does not depend on $U_{n}$ and so we do nothing with this function. We also leave $F_{1}\left(X_{n}, \Omega_{n}, U_{n}\right)$ in its current form at this stage. On the surface defined by (82), we then find that

$$
\begin{aligned}
X_{n+1} & =-\frac{2 c_{2} \Omega_{n}+c_{1}\left(1-X_{n}\right)+\tilde{F}\left(X_{n}, \Omega_{n}\right)}{\left(\beta c_{1}-k\right) \tilde{F}\left(X_{n}, \Omega_{n}\right)}, \\
\Omega_{n+1} & =\frac{\left(5 \beta c_{2}-k c_{3} /\left(2 c_{2}\right)\right) U_{n}}{\beta \tilde{F}\left(X_{n}, \Omega_{n}\right)}, \\
U_{n+1} & =\frac{\left(k-\beta c_{1}\right) U_{n}}{\beta \tilde{F}\left(X_{n}, \Omega_{n}\right)}
\end{aligned}
$$

Taking the limit as $U_{n} \rightarrow 0$ therefore gives $\Omega_{n+1}=U_{n+1}=0\left(\right.$ since $\tilde{F}\left(X_{n}, \Omega_{n}\right)>0$ ) and since $X_{n+1}$ in (83) above does not depend on $U_{n}$, there is no limit to take here.

We now consider conditions on $k$, assuming that $U_{n}<0$ and $0<X_{n}<2$, for this next iterate to lie in $W$. We note that the following analysis does not require $U_{n}$ to be small.

Since $\tilde{F}\left(X_{n}, \Omega_{n}\right)>0$, then $U_{n+1}<0$ provided that

$$
k>\beta c_{1}(>0)
$$

For $X_{n+1}$ defined by (83), we note that the denominator is negative, using (84) and since $\tilde{F}\left(X_{n}, \Omega_{n}\right)>0$. Lemma 7.1 ensures that the numerator of $X_{n+1}$ is positive and combining these results, we conclude that $X_{n+1}>0$ as required.

The final condition we require is that $X_{n+1}<2$. To find the values of $k$ which correspond to the boundary of the region satisfying this condition, we substitute $X_{n+1}=2$ into (83) and solve for $k$, giving

$$
k=\frac{2 c_{2} \Omega_{n}+c_{1}\left(1-X_{n}\right)+\tilde{F}\left(X_{n}, \Omega_{n}\right)}{2 \tilde{F}\left(X_{n}, \Omega_{n}\right)}+\beta c_{1} .
$$

The required inequality is then

$$
k>\frac{2 c_{2} \Omega_{n}+c_{1}\left(1-X_{n}\right)+\tilde{F}\left(X_{n}, \Omega_{n}\right)}{2 \tilde{F}\left(X_{n}, \Omega_{n}\right)}+\beta c_{1}>\beta c_{1} .
$$

Note that the second inequality follows from Lemma 7.1 and since $\tilde{F}\left(X_{n}, \Omega_{n}\right)>0$. Clearly, if this condition holds, then so does (84) and so this is the only condition that we need on $k$ to ensure that the next iterate lies in the region of interest. The surface defined by (82), with $k$ given by the limiting value (85), is the same as was found previously and is given by (59). This is the surface shown in Fig. 8. 


\section{Concluding Remarks}

We have derived an impact map which describes one cycle of the ball from leaving the floor, bouncing on the wall and then bouncing on the floor again. We have analyzed this map in detail to determine some of its properties. This has shown that it is much easier to obtain a higher number of bounces against the wall if the ball is initially thrown away from the wall, but with backspin to bring it back towards the wall after the bounce, than if the ball is initially thrown towards the wall. However, this work still leaves a number of questions:

1. How many hits against the wall are possible? Is it a fixed finite number or can it be arbitrarily large?

2. What happens to the region of initial conditions as the number of hits against the wall increases?

3. How many hits against the wall are possible experimentally?

It is possible to answer the first of these questions from the work that we have done. From Theorem 6.1, in case (iii) there is a stable fixed point and so there will be a large region of initial conditions that are attracted to this fixed point. In this case, the number of iterates in $W$, and hence the number of hits against the wall, can be arbitrarily large (see Fig. 11). However, even in cases (i) and (ii) where this fixed point is linearly unstable, there is a one-dimensional unstable manifold associated with perturbations out of the plane $X_{n}=0$. By taking points closer and closer to the fixed point on this manifold, it is possible to find initial conditions that will give rise to an arbitrarily large number of hits against the wall. However, we note that for the parameter values we have been considering of $\alpha=0.5$ and $\beta=0.9$, the unstable eigenvalue is $\lambda_{1}=24.5481$ and so there is strong repulsion from the fixed point. Thus, the intervals on the unstable manifold which correspond to a given high number of hits will be exceedingly small!

The third question poses an interesting challenge for experimentalists!

\section{Acknowledgements}

The authors thank the referees for a number of useful comments and suggestions which have materially improved the presentation of the work. In particular, one referee supplied us with further references, which have been included in the paper, on alternative mathematical models of the bounce process.

\section{References}

[1] P.J. Aston and R. Shail, The dynamics of a bouncing superball with spin, Dyn. Sys. 22 (2007) 291-322.

[2] R. Garwin, Kinematics of an ultraelastic rough ball, Am. J. Phys. 37 (1969) 88-92.

[3] R. Cross, Measurements of the horizontal coefficient of restitution for a superball and a tennis ball, Am. J.Phys. 70 (2002) 482-489.

[4] R. Cross, Grip-slip behavior of a bouncing ball, Am. J. Phys. 70 (2002) 1093-1102. 
[5] L. Labous, A.D. Rosato and R.N. Dave, Measurements of collisional properties of spheres using high-speed video, Phys. Rev. E 56 (1997) 5717-5725.

[6] H. Dong and M.H. Moys, Experimental study of oblique impacts with initial spin, Powder Tech. 161 (2006) 22-31.

[7] R. Sondergaard, K. Chaney and C.E. Brennen, Measurements of sold spheres bouncing off flat plates, J. Appl. Mech. 57 (1990) 694-699.

[8] N. Maw, J.R. Barber and J.N. Fawcett, The oblique impact of elastic spheres, Wear 38 (1976) 101-114.

[9] W.J. Stronge, R. James and B. Ravani, Oblique impact with friction and tangential compliance, Phil. Trans. R. Soc. Lond. A 359 (2001) 2447-2465.

[10] A. Bibó, Gy Károlyi and T. Bódai, Fly-wheel model exhibits the hither and thither motion of a bouncing ball, Int. J. Nonlin. Mech. 44 (2009) 905-912.

[11] M. Glanville, Experimental investigation of the counter-intuitive back-spin bouncing of a superball between a horizontal floor and a vertical wall, BSc Project, Department of Physics, University of Surrey, 2010.

[12] G.W. Bluman and S. Kumei, Symmetries and Differential Equations, Springer-Verlag, New York, 1989.

[13] W. L. Ferrar, Higher Algebra, Oxford University Press, Oxford, 1950. 


\section{Figure Captions}

Figure 1: The trajectory of the ball centre $C$ and three successive impacts.

Figure 2: (a) Three-dimensional representation of the parameter space $S_{1}$ satisfying $0<$ $\tilde{x}_{0}<-2 \tilde{u}_{0}$. The colours are used to show a grid in the $\tilde{u}_{0}$ and $\tilde{\omega}_{0}$ variables. (b) A trajectory for precisely one rebound from the wall with initial conditions $\tilde{x}_{0}=0.2, \tilde{u}_{0}=-0.5, \tilde{\omega}_{0}=0$. Red indicates negative (i.e. clockwise ball rotation), blue positive and black zero angular velocities. Click for an animation of this case [link].

Figure 3: (a) A three-dimensional representation of the region of parameter space $S_{2}$ satisfying $\tilde{x}_{m}+2 \tilde{u}_{m} \tilde{v}_{m}<0, m=0,1$. (b) The left-hand view of the region depicted in $(a)$. (c) The right-hand view of the region depicted in $(a)$. (d) A typical trajectory with two rebounds from the wall with initial conditions $\tilde{x}_{0}=0.05, \tilde{u}_{0}=-0.2, \tilde{\omega}_{0}=0$. Click for an animation of this case [link].

Figure 4: (a) The set $S_{3}$ : at least 3 impacts on the wall. Note the development of a "depression" on the top left-hand side. (b) The set $S_{4}$ : at least 4 impacts on the wall. (c) The set $S_{5}$ : at least 5 impacts on the wall.

Figure 5: (a) A typical trajectory with three rebounds from the wall with initial conditions $\tilde{x}_{0}=0.025, \tilde{u}_{0}=-0.05, \tilde{\omega}_{0}=0$. Click for an animation of this case [link]. An experiment demonstrating three rebounds from the wall can be seen here [link]. (b) A typical trajectory with four rebounds from the wall with initial conditions $\tilde{x}_{0}=0.29, \tilde{u}_{0}=-0.5, \tilde{\omega}_{0}=-5$. Click for an animation of this case [link].

Figure 6: (a) The set $R_{2}$ : at least 2 impacts on the wall. (b) The set $R_{3}$ : at least 3 impacts on the wall. (c) The set $R_{4}$ : at least 4 impacts on the wall. $(d)$ The set $R_{5}$ : at least 5 impacts on the wall.

Figure 7: The plane $X_{n}=0$ for $\alpha=0.5, \beta=0.9$. In region (a), $X_{n+1}=0, U_{n+1}<0$; in region (b), $X_{n+1}=0, U_{n+1}>0$; in region (c), $X_{n+1} \in(0,2), U_{n+1}<0$ and $X_{n+2} \notin(0,2)$; in region (d), $X_{n+1}, X_{n+2} \in(0,2), U_{n+1}, U_{n+2}<0$; in region (e), $X_{n+1}>2, U_{n+1}<0$; in region (f), $X_{n+1}<0, U_{n+1}>0$. The + symbol indicates the fixed point discussed in Section 6.3.

Figure 8: A contour plot of the surface given by (59) which is defined by $X_{n+1}=2$.

Figure 9: The eigenvalues of $J\left(\Omega_{0}, U_{0}\right)$ in the $(\alpha, \beta)$ plane. (a) The eigenvalues $\lambda_{2,3}$ are complex and $\left|\lambda_{2,3}\right|>1, \lambda_{1}>1$; (b) The eigenvalues $\lambda_{2,3}$ are complex and $\left|\lambda_{2,3}\right|<1, \lambda_{1}>1$; (c) The eigenvalues $\lambda_{2,3}$ are complex and $\left|\lambda_{2,3}\right|<1, \lambda_{1}<1$; (d) The eigenvalues $\lambda_{2,3}$ are real, 
$\lambda_{1}>1$. The solid red line gives parameter values at which $\left|\lambda_{2,3}\right|=1$. The blue line gives parameter values at which the discriminant (69) is zero. The dashed red line gives parameter values at which $\lambda_{1}=1$.

Figure 10: Two projections of the one-dimensional unstable manifold coming from the fixed point in the $X=0$ plane. Also shown is the point where this manifold crosses the plane $X=2$ and two preiterates of this point.

Figure 11: A typical trajectory with $\alpha=0.95, \beta=0.6$ where there are infinitely many bounces of the ball against the wall as the iterates converge to the stable fixed point. The initial conditions are $\tilde{x}_{0}=0.3, \tilde{u}_{0}=-1.2, \tilde{\omega}_{0}=-2.5$. Click for an animation of this case [link].

Figure 12: The blue regions show the parts of the $X_{n}=0$ plane for which $X_{n+k}=0$ and $U_{n+k}<0$ for $k=2,3,4,5$, for $\alpha=0.5, \beta=0.9$. The red plus sign indicates the fixed point.

Figure 13: The red regions show the parts of the $X_{n}=0$ plane for which $0<X_{n+k}<2$ and $U_{n+k}<0$ for $k=2,3,4,5$, for $\alpha=0.5$ and $\beta=0.9$. The red plus sign indicates the fixed point. The boundaries of the regions shown in Fig. 12 are also given.

Figure 14: Regions of the $X_{n}=0$ plane for which $0 \leq X_{n+k}<2$ and $U_{n+k}<0$ for $k=2$ (blue), 3 (red), 4 (green), 5 (magenta), for $\alpha=0.5$ and $\beta=0.9$. The red plus sign indicates the fixed point.

Figure 15: The dashed line shows points at which $j_{11}(\Omega, U)=1$ for $\alpha=0.5, \beta=0.9$. Above the line, $j_{11}(\Omega, U)<1$ and below the line $j_{11}(\Omega, U)>1$.

Figure 16: The line $X_{n}=L_{2}\left(\Omega_{n}\right)$ in the $U_{n}=0$ plane that maps onto the point $\left(\Omega_{n+1}^{(2)}, U_{n+1}^{(2)}\right)$ in the $X_{n+1}=0$ plane. 